\title{
Aufsatz
}

Thomas Freller

\section{Militärische Strategie und christliche Propaganda}

\author{
Der Krieg um Kandia (1645 bis 1669) und der Padre Ottomano
}

DOI 10.1515/mgzs-2017-0001

Zusammenfassung: Im Zuge der erst in den letzten Jahrzehnten auf solide wissenschaftliche Grundlage gestellten Forschungen zum Wesen der Konvertiten und Renegaten geriet auch Osman, später »Padre Ottomano« genannt, - ein angeblicher Sohn von Sultan Ibrahim I. - wieder in das Blickfeld internationaler Aufmerksamkeit. Dabei wurden vor allem seine Gefangennahme durch die Malteserritter im September 1644, der damit geschaffene Vorwand des osmanischen Angriffs auf das damals venezianisch besetzte Kreta und seine folgende Karriere im Dominikanerorden im Kontext des geistigen Klimas der Gegenreformation diskutiert. Die aktive Einbeziehung des Padre Ottomano in militärstrategische Konzepte zu einem Entsatz der von den Osmanen belagerten Hauptstadt Kretas, das damals venezianische Kandia (Candia/Kandiye), sowie seine Tätigkeiten als agent provocateur zur Aufwiegelung der Bevölkerung Griechenlands und des Balkans und damit zur Errichtung einer zweiten Front wurden bisher nicht untersucht. Der vorliegende Beitrag versucht, dieses Desiderat der Forschungen zur Militärgeschichte des Mittelmeers - und durch die Teilnahme bayerischer, braunschweigischer, niedersächsischer und westfälischer Truppenverbände auch Deutschlands - anhand bisher nicht ausgewerteter Archivalien und mittlerweile erschienener Quelleneditionen zu schließen. Dabei wird auch das militärische Scheitern dieser Pläne um den Padre Ottomano im Kontext der englischen, niederländischen und französischen Handelsinteressen diskutiert.

Schlüsselwörter: Osmanisches Reich, Kreta, Kandia, Domenico di San Tommaso, Padre Ottomano, Baldassare Diego Loyola

Kontakt: Thomas Freller, Fachhochschule Aalen (Baden-Württemberg), E-Mail: tfreller@aol.com 


\section{Der Krieg um Kandia in der bisherigen Forschung - Schwerpunkte und Lücken}

Die osmanische Belagerung und Eroberung des zuvor venezianisch beherrschten Kreta, der sogenannte Krieg um Kandia ("guerra di Candia «) 1645-1669, und seine internationalen Auswirkungen wurden bisher von verschiedener Seite aufgearbeitet. Autoren wie Ekkehard Eickhoff, Kenneth Meyer Setton, Stanford J. Shaw/Ezel Kural Shaw, Molly Greene, Dorothy M. Vaughan, Guido Candiani, Özkan Bardakçi und François Pugnière beschäftigten sich mit den politischen Implikationen des Krieges, ${ }^{2}$ Roger Charles Anderson, Alan G. Jamieson, Arrigo Petacco, Wilhelm Kohlhaas und Klaus-Peter Matschke konzentrierten sich auf maritime und militärische Aspekte. ${ }^{3}$ Vor allem die italienische Forschung hat sich mit der Rolle der an den verschiedenen Entsatzunternehmungen gegen die osmanischen Belagerer an führender Position beteiligten Offiziere bzw. Admiräle Francesco Morosini (der 1688 zum Dogen gewählt werden sollte), Geronimo Francesco Villa, Antonio Barbaro und Bernardo Nani beschäftigt. ${ }^{4}$ Dabei geriet auch die Beteiligung deutscher, österreichischer und schweizerischer Kontin-

1 In den zeitgenössischen italienischen Archivalien erscheinen Kreta und seine Hauptstadt als "Candia«. In den osmanischen Dokumenten wird »Kandiye« benutzt. Hier wird auf den in den zeitgenössischen deutschen Quellen erscheinenden Begriff »Kandia« zurückgegriffen.

2 Kenneth Meyer Setton, Venice, Austria, and the Turks in the Seventeenth Century, Philadelphia, PA 1991, S. 110-115; Ekkehard Eickhoff, Venedig, Wien und die Osmanen: Umbruch in Südosteuropa 1645-1700, Stuttgart 2009, S. 27-32; Molly Greene, A Shared World. Christians and Muslims in the Early Modern Mediterranean, Princeton, NJ 2000, S. 62-64 und S. 110-113; Dorothy M. Vaughan, Europe and the Turk, Liverpool 1954, S. 256-259; Guido Candiani, Francia, Papato e Venezia nella fase finale delle guerra di Candia. In: Atti dell'Istituto Veneto di Scienze, Lettere ed Arti (Classe di Scienze Morali, lettere ed arte), 152 (1993-1994), 4, S. 829-887; La Dernière Croisade. Ed. par Özkan Bardakçi et François Pugnière, Rennes 2008; Stanford J. Shaw and Ezel Kural Shaw, History of the Ottoman Empire and Modern Turkey, 2 vols., Cambridge 1973, Bd 1, S. 207-215. Auf einige der zahlreichen sich innerhalb von Abrissen der Geschichte des Osmanischen Reiches und Venedigs mit dem Krieg um Kandia beschäftigenden Autoren wird in den Fußnoten 6, 7 und 12 verwiesen.

3 Arrigo Petacco, L'ultima crociata. Quando gli Ottomani arrivarono alle porte dell'Europa, Mailand 2009, S. 123-135; Alan G. Jamieson, Lords of the Sea: A History of the Barbary Corsairs, London 2012, S. 127-131; Roger Charles Anderson, Naval Wars in the Levante 1559-1853, Liverpool 1951, S. 121-132; Wilhelm Kohlhaas, Candia 1645-1669. Die Tragödie einer abendländischen Verteidigung mit dem Nachspiel Athen 1687, Osnabrück 1978; Klaus-Peter Matschke, Das Kreuz und der Halbmond. Die Geschichte der Türkenkriege, Düsseldorf, Zürich 2004, S. 368-375.

4 Vgl. Andrea Da Mosta, I Dogi di Venezia nella vita pubblica e privata, Florenz 1977, S. 427-480; Alessandro Marzo Magno, Atene 1687, Mailand 2011, S. 41-48, und die Passagen in Lucette Valensi, The Birth of the Despot: Venice and the Sublime Port, Ithaca 1993. 
gente, namentlich die Entsendung von Einheiten unter Josias von Waldeck, Heinrich Ulrich Graf von Kielmansegg, Adrian Leopold von Bühren, Stephan von Closen, Wilhelm von Forstenau, Johann Werdmüller und anderer, in das Blickfeld. ${ }^{5}$

Bisher wurde von der Forschung allerdings nicht der strategische Einsatz einer damals in der christlich-okzidentalen Welt sehr populären Figur, des Dominikanerpaters Domenico di S. Tommaso bzw. Padre Ottomano, im Konzept eines militärischen Entsatzes Kretas berücksichtigt. Zwar finden sich bei einigen Autoren kurze Anmerkungen zu seiner Anwesenheit auf Kreta in der Endphase des Krieges, die tieferen Gründe seiner Reise von Paris in das östliche Mittelmeer und seine Aktivitäten zur Errichtung einer zweiten Front gegen die Osmanen wurden dabei von der Forschung kaum beleuchtet. ${ }^{6}$ Die Archivalien und einige Quelleneditionen geben jedoch nähere Einblicke in die militärstrategischen Hintergründe. Der vorliegende Beitrag versucht, anhand der Quellen in den Archiven des Johanniter- und Dominikanerordens, einiger von der Forschung

5 Josias von Waldeck starb an seinen Verwundungen auf Kreta am 9.8.1669; vgl. ausführlich Militärische Mitteilungen, 2 (1829), 1, S. 1-63; 2 (1829), 3, S. 215-259; Bernhard Erdmannsdörffer, Deutsche Geschichte vom Westfälischen Frieden bis zum Regierungsantritt Friedrichs des Großen, 1648-1740, Bd 1, Paderborn 2015 (Nachdr. der Originalausgabe von 1892), S. 523f. Die bayerischen Entsatztruppen standen unter dem Kommando von Adrian Leopold von Bühren, zu seinen Berichten vgl. Joseph Würdinger, Antheil der Bayern an der Vertheidigung Candias 1645-1669. In: Sitzungsberichte der bayerischen Akademie der Wissenschaften, phil.-hist. Klasse 6 (1880), S. 314-347. Zur Rolle Waldecks, der Entsendung kurbayerischer Truppen unter Oberstleutnant von Bühren und der Beteiligung der deutschen Offiziere Erbach, Seebach, Raeßfeld, Ehrenreuter und Styrum vgl. auch: Das lange bestrittene Königreich Candia. Hrsg. von Matthaeus Merian, Frankfurt a.M. 1670, S. 31-75; Geschichte des kurbayerischen Heers insbesonders unter Ferdinand Maria 1651-1679, Bd 1. Hrsg. von Karl Staudinger, München 1901, S. 255-262. Der Schweizer Johann Werdmüller diente als Kommandant der Artillerie unter Ghiron di Ville, Befehlshaber der Kontingente des Herzogs von Savoyen. Vgl. François Savinien d'Alquié, Les Mémoires du Voyage de Ghiron François Marquis de Ville au Levant, ou l'Histoire du Siège de Candie, Amsterdam 1671.

6 Der sich auf Girolamo Brusoni (Historia dell'ultima Guerra trà Veneziani et Turchi, Venedig 1673, Kap. XXIV, S. 222) und das in seinem Besitz befindliche türkische Manuskript eines Offiziers von Ahmet Köprülü (»Dschewahiret-tewarich«, 481 Blätter, hier Bl. 364f.) stützende Joseph von Hammer-Purgstall (Geschichte des Osmanischen Reiches, Bd 6, Budapest 1830, S. 238) schreibt: »Diesen Verlust ersetzte der venezianischen Seemacht keineswegs die Ankunft der päpstlichen Hülfsgaleeren, unter dem Befehle Rospigliosi's, des Neffen Papst Clemens IX., welcher aber statt Hülfstruppen, nur den Dominikaner Padre Ottomano, den angeblichen Bruder Mohammed's IV. am Bord hatte. Auf dem türkischen Pilgerschiffe, dessen Wegnahme den Ursprung des kretischen Krieges, gefangen genommen, und sogleich für einen Sohn Ibrahim's ausgegeben, erschien er nun abermahl am Ende des kretischen Kriegs auf Kreta, ohne dass seine Unbedeutentheit den Begebenheiten, oder diese demselben grösseren Schwung zu geben vermochten.« 
wenig berücksichtigter zeitgenössischer Druckwerke und vor wenigen Jahren erschienener Quelleneditionen zu rekonstruieren, inwiefern Padre Ottomano »instrumentalisiert « wurde, um die Belagerung aufzuheben und den gesamten osmanisch dominierten griechischen Raum zu destabilisieren.

Zum besseren Verständnis dieser Vorgänge erscheint zunächst ein näherer Blick auf die Vorgeschichte der Gefangennahme des Protagonisten angebracht. Dieses Ereignis wurde bereits von zahlreichen zeitgenössischen Chronisten und modernen Historikern mehr oder weniger ausführlich erwähnt und kommentiert. ${ }^{7}$

\section{Prolog}

Am 28. September 1644 attackierten sechs Galeeren der Malteserritter unter dem Kommando von Generalkapitän Gabriel de Chambres Boisbaudrant in der Nähe von Rhodos einen Konvoi osmanischer Schiffe. Dieser Konvoi befand sich auf dem Weg von Konstantinopel nach Alexandria und bestand aus zehn Schiffen; zusammengesetzt aus Pinken und Schaiken und einer größeren Galeone, in den zeitgenössischen Quellen als »gran galeone« oder »gran sultana « bezeichnet. ${ }^{8}$

7 Bartolomeo Dal Pozzo, Historia della Sacra Religione militare di S. Giovanni Gerosolimitano detta di Malta, 2 vol., Verona 1703-1715, hier vol. 2, S. 91; Richard Knolles and Paul Rycaut, Generall Historie of the Turkes, London 1700, vol. 2, S. 56; Amy A. Bernardy, L'incidente di Malta (1644-1645) e il Padre Ottomano. In: Archivio Storico di Malta, 7 (1936), S. 449-462, hier S. 452-454; Ramiro Vadalà, Il Padre Ottomano. In: Malta Letteraria, 1 (1904), 2, S. 47-50; Salvatore Salomone-Marino, La cattura del galeone Gran Sultana. In: Archivio Storico Siciliano. Neue Serie, 22 (1897), S. 238-247, hier S. 238-240; Carl Peter Berly, Kern der Osmanischen Reichsgeschichte, Leipzig 1837, S. 213. Zu den Ereignissen des Septembers 1644 und zum weiteren Schicksal Osmans vgl. auch René Jean Durdent, Beautés de l'histoire di Turquie, Paris 1816, S. 315; Clarence Daniel Rouillard, The Turk in French History, Thought, and Literature, Paris 1938, S. 97; Henry de Castries, Les Sources inédites de l'histoire du Maroc, première série, II/1 Paris 1922, S. 203-240, zu Osman vgl. S. 205, 207.

8 Die Ereignisse vom 28.9.1644 werden in verschiedenen Manuskripten ausführlich beschrieben; vgl. Archive of the Order of Malta (AOM), Valletta, Malta, Ms. 257, ff. 183-196 (»Relatione del Cavre. Verdille sopra la perdità od abandon del galeone preso dalle galera lette nel Ven. Consiglio sotto li 21 di Decembre 1644«); AOM, Ms. 1759, ff. 224r-v (Bericht von Nicolas Cotoner über die Eroberung der gran galeone); AOM, Ms. 1769, ff. 79r-92r (»Relatione del sanguinoso combattimento, e presa d'un galeone e d'un pinco de Turchi fatta dale galere di Malta all crociere di Rodi, il di 28 Settembre 1644«), ff. 97r-101r; Biblioteca Vaticana, Codice Ottoboniano 2688, ff. 6-13 (»Relatione della Gloriosa Vitoria delle Galere di Malta, d'un Gran Galeone, et un grosso Pinco de Turchi sopra le croceriere di Rodi, alli 28 Settembre 1644«) und den Bericht in der Biblioteca Comunale of Palermo, Miscellanea Qq. E 16, Nr. 5. Die Ereignisse fanden ferner ein vielfältiges Echo in der historiografischen, poetischen und kulturwissenschaftlichen Literatur des ausgehenden 17. und 18. Jahrhunderts. Vgl. stellvertretend Madelaine-Angélique de Gomez [u.a.], 
Nach einem fünfstündigen Kampf und hohen Verlusten auf beiden Seiten gelang es den Maltesern, die Galeone und eine Schaike zu erobern sowie eine Pink zu versenken. Die Verluste der Malteser betrugen 116 Soldaten, Seeleute und Galeerenruderer; 11 Ordensritter und 251 Soldaten wurden verwundet. Unter den 380 Gefangenen befanden sich neben verschiedenen höher gestellten Mekka-Reisenden auch der "gran judice del Cairo« Muhammad Effendi aus Bursa und eine Dame aus dem Harem des Sultans mit ihrem etwa zweieinhalbjährigen Sohn. ${ }^{9}$ Die Beute und Gefangenen wurden nach einem Zwischenstopp im Hafen von Kalismene an der Südküste Kretas nach Malta gebracht.

In den nächsten Monaten und Jahren verbreiteten sich von Malta ausgehend Gerüchte, bei der Haremsdame, genannt Zaphira/Zafira, handele es sich um die Favoritin Sultans Ibrahim I. und bei dem Jungen um Osman, den erstgeborenen Sohn des Sultans. ${ }^{10}$

Anecdoti, osia, la storia segreta della Famiglia Ottomana, Neapel 1736, S. 378-381; Vincenzo Abbondanza, Dizionario Storico delle vite di tutti Monarchi Ottomani, Rom 1786, S. 81-83. Ebenso finden sich Hinweise in den sich mit der Geschichte des frühneuzeitlichen Venedigs und Osmanischen Reiches beschäftigenden Abhandlungen. Vgl. Vincent Mignot, The history of the Turkish or Ottoman Empire, from its foundation in 1300, to the peace of Belgrade in 1740, vol. 3, London 1787, S. 97; Clarence Daniel Rouillard, The Turk in French History, Thought, and Literature, Paris 1938, S. 97; Alexandre-Louis-Félix Alix, Précis de l'histoire de L’Empire Ottoman, t. 2, Paris 1822, S. 92f. Auch neuere Untersuchung zum Sklaven- und Renegatenwesen erwähnen das Ereignis, vgl. Giovanna Fiume, Schiavitù mediterranee. Corsari, rinnegati e santi di età moderna, Mailand 2009, zu Baldassare Diego und Padre Ottomano vgl. S. 274-276.

9 Über das Alter des Kindes gibt es verschiedene Angaben. Aldighero Fontana (L'Origine della Sacra et Eminentissima Religione Gerosolimitana, Bologna 1704, S. 336) schreibt über einen "fanciullo di cinque anni, d'aria nobile, e maestosa, e correva voce che fosse uno d' figliuoli del Sultano Ibraimo, spedito del medesimo con la madre in Arabia. « Der Großteil der Autoren gibt das Alter mit zwei bis drei Jahren an. Vgl. Dal Pozzo, Historia della Sacra Religione (wie Anm. 7), vol. 2, S. 91; Knolles/Rycaut, Generall Historie (wie Anm. 7), vol. 2, S. 56.

10 Vgl. die Monografien von Ottaviano Bulgarini, Vita del Padre Mestro F. Domenico di S. Tomaso dell'Ordine de' Predicatori, ditto prima Sultan Osman Ottomano, figlio d'Ibraim Imperador de' Turchi, Neapel 1689, auch ebd. 1698 und 1708, und französische Übersetzungen; Jacques de Jant, L'Histoire du reverend Père Dominique Ottoman, de l'Ordre des FF. Prescheurs, sous le nom du Prince Osman, fils du Sultan Ibrahim, Empereur des Turcs, Paris 1665; Felice Tempia, Compendio della vita del P. Domenico di S. Tommaso ditto Ottomano, Turin 1757; Thomas Freller and Dolores Campoy Felices, Padre Ottomano and Malta. A Story of the 1001 Nights, Malta 2006, und die Zusammenfassung in Daniele Callus, Il P. Domenico Ottomano (Fu vero principe?). Saggio storico-critico, Rom 1918. Zum Tod seiner nur wenige Monate nach der Ankunft auf Malta verstorbenen Mutter Zaphira vgl. Corrispondenza tra la Corte di Roma e l'inquisitore di Malta durante la Guerra di Candia (1645-1669). Hrsg. von Paolo Piccolomini. In: Archivio Storico Italiano, Serie V, 41 [1910], p. 2, S. 28. Für einen osmanischen Blickwinkel vgl. Musa Kılıç, Osmanlı tahtında hak iddia eden bir maceraperest: Düzme şehzade Ahmet Nadir Bey. An Adventurer who asserted right on the Ottoman Throne: Pretender Prince Ahmed Nadir Bey. In: Interna- 
Der Junge Osman ist untrennbar mit dem 1645 beginnenden »Krieg um Candia« verknüpft. Vermutlich diente der Angriff auf die »gran galeone«, die Gefangennahme ihrer hochrangigen Passagiere und speziell der Aufenthalt des maltesischen Verbandes im Hafen von Kalismene als Vorwand für Sultan Ibrahim I., Venedig den Krieg zu erklären. ${ }^{11}$ Im Juni 1645 begann er einen schon länger vorbereiteten Angriff auf Kreta. ${ }^{12}$ Bereits in der Sommerkampagne des Jahres 1645 eroberten die Osmanen die zweit- und drittgrößte Stadt der Insel, Chania und Rethymnon, und einen Großteil des Landesinneren. ${ }^{13}$

Nach dem frühen Tod der Mutter im Winter 1644/45 blieb der junge Osman auf Malta. ${ }^{14}$ Der portugiesische Ordensritter Raimondo d'Alvito wurde von Großmeister Lascaris Castellar zum Tutor und Vormund des jungen Türken eingesetzt. 1656 wurde in einer glanzvollen Zeremonie die Taufe Osmans begangen. ${ }^{15}$ In den nächsten Monaten wurde er in den Dominikaner-Orden aufgenommen und trug von nun an den Namen Domenico di S. Tommaso. Diese Konversion und Karriere

tional Journal of Social Science, 5 (2012), 6, S. 329-337, zu Padre Ottomano vgl. S. 329f. Porträts von »Hosmanus Turcarum Imperatoris Ibrahim Filius" und seiner Mutter "Sultana Zaffira" sind abgedruckt in: Das lange bestrittene Königreich Candia (wie Anm. 5), S. 4.

$11 \mathrm{Zu}$ den osmanischen Vorbereitungen für den Angriff auf Kreta vgl. die Briefe Giovanni Soranzos an den venezianischen Senat vom 11.1. sowie 5. und 13.3.1645, Archivio di Stato di Venezia, Senato, dispacci ambasciatori, Serie 127; Public Record Office, London, Calendar of State Papers, »Venetian«, xxviii, rub. 190, hier zit. nach Victor Mallia-Milanes, Venice and Hospitaller Malta. 1530-1798: Aspects of a Relationship, Malta 1992, S. 160; Eickhoff, Venedig, Wien und die Osmanen (wie Anm. 2), S. 27-31 (zu Padre Domenico di S. Tommaso, vgl. auch S. 425); Heinrich Kretschmayr, Geschichte von Venedig, Bd 3, Stuttgart 1934, S. 313-315 (zur Seeschlacht vom 28.9.1644), S. 333f. (zum Krieg um Kandia, ohne Verweise auf den Padre Ottomano); Nicolae Jorga, Geschichte des Osmanischen Reiches, Bd 4, Paderborn 2015 (Nachdruck des Originals von 1913), S. 122-134; Petacco, L'ultima crociata (wie Anm. 3), S. 123-125; Jamieson, Lords of the Sea (wie Anm. 3), S. 127-129.

12 Vgl. auch die neueren Untersuchungen von Luigi Robuschi, Il Rapporto tra Repubblica di Venezia e l'Ordine di Malta e ele relazioni economiche tra sei e settecento, Diss., Padua 2012, S. 76-82; die ältere und neuere Literatur befasst sich aus den verschiedensten Blickwinkeln mit dem osmanischen Angriff auf Kreta, vgl. Jean Baptiste Rostagne, Histoire curieuse du Siege du Candia, Amsterdam 1671, S. 18-25; Pierre-Antoine-Noël-Bruno Duc de Daru, Geschichte der Republik Venedig, Bd 3, Leipzig 1854, S. 324-326; Samuele Romanin, Storia documentata di Venezia, vol. 7, Venedig 1857, S. 246-249; Vaughan, Europe and the Turk (wie Anm. 2), S. 256-260; Wilhelm Bigge, Der Kampf um Candia in den Jahren 1667-1669, Berlin 1899 (= Kriegsgeschichtliche Einzelschriften, 26), S. 115-117; Mario Monterisi, L'Ordine a Malta, Tripoli e Italia, Mailand 1940, vol. 2, S. 76-78 und S. 139.

13 Vgl. Greene, A Shared World (wie Anm. 2), S. 62-64 und S. 110f.; Anderson, Naval Wars in the Levante (wie Anm. 3), S. 121-123.

14 Dal Pozzo, Historia della Sacra Religione (wie Anm. 7), vol. 2, S. 244.

15 Ebd. 
in einem christlichen Orden stellte einen der großen Triumphe der Gegenreformation dar und wurde in verschiedensten Pamphleten und Drucken gefeiert. Stiche mit Padre Ottomanos Porträt zirkulierten und unterstrichen die Popularität des Konvertiten. ${ }^{16}$ Dabei zweifelten die katholischen Medien zunächst nicht an, ob Domenico di S. Tommaso alias Padre Ottomano wirklich der leibliche Sohn von Sultan Ibrahim I. war. Für den vorliegenden Beitrag ist es von untergeordneter Bedeutung, ob dies der Wahrheit entsprach oder nicht. ${ }^{17}$ Festzuhalten ist lediglich, dass die Mehrheit der Bevölkerung in den Staaten Italiens, in Spanien, Frankreich oder Deutschland in Padre Ottomano einen echten Sohn des Sultans sah. ${ }^{18}$

\section{Missionsarbeit und politische Strategie}

Den Anstoß, Padre Ottomano in einer gegen das Osmanische Reich gerichteten militärischen Mission einzusetzen, scheinen die Erfolge eines anderen zeitgenössischen Konvertiten gegeben $\mathrm{zu}$ haben. Die Rede ist von dem ebenfalls von Verbänden des Johanniterordens nach Malta verschleppten Sohn des Fürsten Abdelwahid von Fez, Muhammad al-Attaz (in christlichen Quellen »Mehmed bin Thesi ). ${ }^{19}$ Nachdem er im Juli 1656 auf Malta getauft worden war, ${ }^{20}$ übernahm er

16 Vgl. die Abb. in Freller/Campoy Felices, Padre Ottomano and Malta (wie Anm. 10), S. 47 und S. 54.

17 Vgl. die Untersuchungen von Callus, Il P. Domenico Ottomano (wie Anm. 10); Bernardy, L'incidente di Malta (wie Anm. 7). Ausführlich auch Freller/Campoy Felices, Padre Ottomano and Malta (wie Anm. 10).

18 Vgl. Bulgarini, Vita del Padre (wie Anm. 10); De Jant, L'Histoire du reverend Père Dominique Ottoman (wie Anm. 10); Theatrum Europaeum, Bd 5. Hrsg. von Erasmus Francisci, Nürnberg 1690, S. 842; Christoph Boetius, Glanzerhöhter und Triumph leuchtender Kriegshelm, Bd 3, Nürnberg 1688, S. 268 (mit Porträtbildern); Das lange bestrittene Königreich Candia (wie Anm. 5), S. 4-7 (mit Porträtbildern); Neu-vermehrtes historisch- und geographisches allgemeines Lexicon, T. 5. Hrsg. von Jakob Christoph Beck, August Johann Buxtorff und Jakob Christoph Iselin, Basel 1744 , S. $510 f$.

19 In der zeitgenössischen Literatur erscheint er als Mehmed oder Mehmet Bin Thesi, vgl. Dal Pozzo, Historia della Sacra Religione militare (wie Anm. 7), hier vol. 2, S. 189: „Generale Demandolx con 4 galere [...] arrestò 32 schiavi [...] Capo Buono, sopra un vascelo Inglese [...] frà i quali [...] Mehemet Bin Thesi, figlio del Re de Fes, e di Marocco, giovine di 21 anni.« Zu Mehmet Ben Thesi vgl. auch An Universal History from the earliest accounts to the present time, vol. 15. Ed. by George Sale [u.a.], London 1781, S. 440.

20 Vgl. Baldassare Diego Loyolas Autobiografie »Notizie Spettanti alla vita del Padre Baldassare Loyola Mandes«, aufbewahrt im Archivio della Pontificia Università Gregoriana (APUG), Rom, 1060, i/ii, f. 32-48; Dal Pozzo, Historia della Sacra Religione (wie Anm. 7), vol. 2, S. 244. Zur 
die Namen seiner Tutoren, der Ritter Baldassare Demandolx und Diego de Melo. Von nun an nannte er sich Balthasar Diego bzw. italienisch Baldassare Diego. Zu Ehren des Heiligen Ignatius von Loyola, dessen Namenstag sein Tauftag war, wählte er den Nachnamen »Loyola«.

Baldassare Diego Loyola siedelte nach Sizilien über, wo er in den Jesuitenorden aufgenommen wurde. 1661 begab er sich für sein Noviziat nach Rom; zwei Jahre später erfolgte seine Weihe zum Priester. ${ }^{21}$ In den Jahren 1664 bis 1667 widmete er sich mit bemerkenswertem Erfolg der christlichen Missionsarbeit in den Häfen von Genua und Neapel. Anhand seiner Notizen lassen sich nicht weniger als 880 von ihm bewirkte Konversionen von muslimischen Seeleuten, Soldaten und Galeerensklaven nachweisen. ${ }^{22}$

Sein Status als ehemaliger Prinzensohn und seine Karriere in der Societas Jesu brachten Loyola in Kontakt mit führenden Mitgliedern der Römischen Kurie, u.a. den Kardinälen Antonio Barberini und Benedetto Odescalchi (dem zukünftigen Papst Innozenz XI.) sowie mit Mitgliedern des Fürstenhauses von Savoyen, der Medici und der Doria. Auf seinen Reisen traf er in Turin auch Padre Ottomano. Den Angaben Loyolas zufolge verlief der Austausch mit jenem sehr kollegial. ${ }^{23}$

Sein weiteres Leben ist für das Thema dieses Beitrags nicht von Belang. Nachdem der Jesuitengeneral für Missionstätigkeiten in Nordindien seine Zustim-

Konversion und seiner späteren Vita vgl. ausführlich Tirso Gonzalez, Manuductio ad Conversionem Mahumetanorem, Bd 2, Dillingen 1688-1689, S. 53-58 (= Kapitel »Mirabilis conversion Regis de Fez, postea Balthasar de Loyola Societas Jesu «); Patrice Sanguy, Le père de Mandes, un prince Maroccain chez les jésuites, Mémoire. In: Les cahiers d'Afrique du Nord, 83 (2013), S. 80-99; Emanuele Colombo, A Muslim turned Jesuit: Baldassare Loyola Mandes (1631-1667). In: Journal of Early Modern History, 17 (2013), 5/6, S. 479-504; Emanuele Colombo, Baldassare Loyola de Mandes (1631-1667), prince de Fez et jésuite. In: Les Musulmans dans l'histoire de l'Europe, t. 1. Ed. par Bernard Vincent et Jocelyn Dakhlia, Paris 2011, S. 159-193; vgl. auch die älteren Beiträge von Paul Duclos, Baldassare Loyola Mandes. In: Diccionario Histórico de la Compañia de Jesús: biográfico-temático. Ed. di C.E. O’Neill e J.M. Dominguez, Rom, Mailand 2001, S. 2428; Carlos García Goldáraz, Un principe de Fez Jesuita: Scheik Muhammad Attasi, en religion P. Balthasar Diego Loyola de Mandes (1631-1667): Estudio sobre su ascendencia rega, Burgos 1944; Louis Lebessou, La seconde vie d'un sultan du Maroc. In: Etudes, 123 (1910), S. 488-498.

21 Corrispondenza (wie Anm. 10), S. 12 und S. 19; Dal Pozzo, Historia della Sacra Religione (wie Anm. 7), vol. 2, S. 116-118, 186 und S. 190.

22 Vgl. »Notizie Spettanti alla vita del Padre Baldassare Loyola Mandes«, APUG, Rom, 1060, i/ii, f. 221-225; vgl. auch Peter M. Mazur, Combating "Muhammedan Indecency«: The Baptism of Muslim Slaves in Spanish Naples, 1563-1667. In: Journal of Early Modern History, 13 (2009), S. 25-48, und die Verweise in Salvatore Bono, Schiavi musulmani nell'Italia moderna. Galeotti, vù comprà, domestici, Neapel 1999.

23 Vgl. De Castries, Les sources inédites (wie Anm. 7), S. 207. 
mung erteilt hatte, bereitete Loyola seine Reise vor, starb jedoch bereits am 15. September 1667 auf dem Weg nach Lissabon im Colegio Imperial von Madrid. Unter anderem auf Betreiben der Regentin Mariana von Österreich erschienen in den nächsten Monaten verschiedene, Loyolas vorbildliches Leben verherrlichende Sonette und Monografien ${ }^{24}$. In Pedro Francisco Esquexs »Sermon fúnebre historial « ${ }^{25}$ (1667) erscheint er als ein neuer Heiliger Paulus, der wie der Apostel den Weg vom Ungläubigen zum unermüdlichen Missionar beschritten hatte.

Es waren höchstwahrscheinlich Loyolas Erfolge in der Missionsarbeit, die Vertreter der Römischen Kurie und auch Venedigs dazu veranlassten, den noch populäreren und als gebürtiger Osmane geeigneteren Domenico di S. Tommaso alias Padre Ottomano für militärische Zwecke im östlichen Mittelmeer einzusetzen. ${ }^{26}$ Dieses Projekt musste angesichts des in seine entscheidende Phase tretenden Krieges um Kandia noch dringlicher erscheinen.

\section{Der Padre Ottomano und Kreta - Einsatzpläne zwischen Visionen und Realitäten}

Während sich im Sommer 1667 Loyola auf seine Missionsreise nach Indien vorbereitete, wurden in Rom und Venedig Pläne für einen Kreta-Einsatz des Padre Ottomano diskutiert. Padre Ottomano lebte zu dieser Zeit nach einer triumphalen

241692 verfasste Loyolas Tutor, der Direktor des Collegium Romanum, Domenico Brunacchi, eine ausführliche Biografie des Konvertiten, wahrscheinlich um die Kanonisierung seines früheren Schützlings zu befördern, vgl. „Vita del Ammirabilie P. Baldassare Loiola de Mandes della Compagnia di Gesù: Prodigio della Divina Grazia«. Das nicht veröffentlichte Manuskript befindet sich im Archivum Romanum Societatis Jesu, Rom, Vitae $105 \mathrm{f}$.

25 Pedro Francisco Esquex, Sermon fúnebre historial en las exepuias que se celebrant en el Colegio Imperial de la Compañia de Jesús al Venerable P. Balthasar de Loyola Mandez, Madrid 1667.

26 Diese Überlegungen und Pläne mögen auch durch die Erinnerung an den nach 1614 auf dem Balkan aktiven sogenannten Il Sultano Jachio Ottomano motiviert worden sein. Auch dieser angebliche Sultanssohn war an Verhandlungen zwischen politischen Rebellenführern auf dem Balkan und westlichen Fürsten beteiligt. Vgl. Amanda Danielle Giammanco, (Self)Fashioning of an Ottoman Christian Prince: Jachia ibn Mehmed in confessional diplomacy of the early Seventeenth-Century, unpublizierte Magisterarbeit, Central European University Budapest 2015, S. 34-40; Vittorio Catualdi, Sultan Jahya della casa imperiale ottomana ed altrimenti Alessandro Conte di Montenegro ed i suoi discendenti in Italia, Triest 1889. 
Tour d'Europe an verschiedenen italienischen Fürstenhöfen zurückgezogen im Konvent von SS. Annunziata in Paris.

Die internationale, militärische und logistische Hilfe für das belagerte Kandia durch den Heiligen Stuhl, Florenz, Genua, den Malteserorden oder deutsche Fürstentümer setzte erst verzögert ein. Dies lag zum einen an den komplexen logistischen Herausforderungen bei der Rekrutierung sowie beim Transport der Truppen und des schweren Geräts nach Venedig und von dort über die Adria nach Kreta, zum anderen an den vorhergehenden politischen Spannungen zwischen der Serenissima und einigen der Truppen entsendenden Mächte, etwa mit dem Malteserorden oder der Republik Genua. ${ }^{27}$

Besonders die Situation der aufstrebenden Großmacht Frankreich war in diesem Kontext ambivalent. Die französisch-osmanischen Beziehungen blieben auch im 17. Jahrhundert nicht nur aus ökonomischen Gründen eng; die Konflikte mit der Dynastie der sowohl in Spanien als auch in Österreich regierenden Habsburger bedeuteten ein einendes Moment für Paris und Konstantinopel. ${ }^{28}$ Diese Situation sollte auch aufgrund der im Zuge der osmanischen Expansionsbestrebungen 1683 hergestellten Allianz zwischen den habsburgischen Ländern, Venedig, Polen, dem Kirchenstaat und Russland weiter fortbestehen. ${ }^{29}$ Dass ungeachtet der guten Beziehungen des Hofes Ludwigs XIV. zur Hohen Pforte die Fraktionen der französischen Gesellschaft unterschiedliche Haltungen gegenüber den Osmanen einnahmen, zeigt die Beteiligung teilweise privat finanzierter Kontingente bei den Entsatzversuchen des belagerten Kandia.

Der Krieg um Kandia wurde mit der Ermordung Sultan Ibrahims I. und der Thronbesteigung des eher trägen Mehmet IV. 1648 nicht beendet. Die 1650er Jahre waren von einigen spektakulären maritimen Erfolgen der vereinigten

27 Zur Unterstützung des Ordens vgl. Dal Pozzo, Historia della Sacra Religione (wie Anm. 7), vol. 2, S. 76-80; Ettore Rossi, Storia della Marina dell'Ordine di S. Giovanni, di Rodi e di Malta, Rom, Mailand 1926, S. 70f.; Corrispondenza (wie Anm. 10), 41 (1908), p. 2, S. 9-12, 45 (1910), 46 (1912).

28 In diesem Kontext vgl. Gábor Ágoston, Information, Ideology, and Limits of Imperial Policy: Ottoman Grand Strategy in the Context of Ottoman-Habsburg Rivalry. In: The Early Modern Ottomans: Remapping the Empire. Ed. by Virginia Aksan [u.a.], New York 2007, S. 75-103.

29 Diese Entwicklung der osmanisch-französischen Beziehungen können nachvollzogen werden anhand der von Ernest Charrière publizierten Dokumente: Négociations de la France dans le Levant, ou, Correspondances, mémoires et acted diplomatiques des ambassadeurs de France à Constantinople et des ambassadeurs, envoyés ou résidents à divers titres à Venise, Raguse, Rome, Malte et Jérusalem, en Turquie, Perse, Géorgie, Crimée, Syrie, Egypte, etc., et dans les états de Tunis, d'Alger et de Maroc, 4 t., Paris 1848-1860; zu den zeitgenössischen diplomatischen Kontakten vgl. auch Maria Pia Pedani, In Nome del Gran Signore: Inviati Ottomani a Venezia dalla Caduta di Costantinopoli alla Guerra di Candia, Venedig 1994. 
venezianischen, florentinischen, päpstlichen und maltesischen Flotten geprägt, die jedoch keinen entscheidenden Durchbruch in der Aufhebung der Belagerung und Blockade der wichtigsten Häfen Kretas brachten. Die christliche Strategie war es, die osmanische Flotte und Transportverbände bereits an der Mündung der Dardanellen in Gefechte zu verwickeln bzw. zu blockieren. ${ }^{30} 1651$ bei Naxos und 1656 in den Dardanellen erlitt die osmanische Marine empfindliche Niederlagen, ${ }^{31}$ dennoch war bereits 1650 außer der Hauptstadt Kandia (heute Iraklio) und den Festungen Gramvousa (ital. Carabusa), Kalydon (ital. Spinalonga), Sitia und Souda fast die gesamte Insel von den Osmanen besetzt oder kontrolliert.

Die Jahre bis 1667 können als »eingefrorener« Krieg bezeichnet werden, der massive Einsatz von osmanischen Truppen auf dem Balkan, in Rumänien und Ungarn sowie an der persischen Grenze verhinderte eine aktive und energische Fortsetzung der Belagerungen der kretischen Festungen. ${ }^{32}$ Ungeachtet des Einsatzes von Kontingenten aus Deutschland, Savoyen, den Niederlanden und Frankreich konnte dennoch ein nachhaltiger Entsatz der Festungen nicht erreicht werden.

Als 1664 und 1666 Großwesir Ahmet Köprülü̈33 - damals die entscheidende Persönlichkeit in der osmanischen Politik - Waffenstillstandsabkommen bzw. Friedensverträge mit Kaiser Leopold I. schloss, konnten frische Truppen nach Kreta verlegt werden. Dorthin führte im Herbst des Jahres 1666 Ahmet Köprülü persönlich Truppenteile. In der nun folgenden Kampagne wurden weitere venezianische Stützpunkte erobert. Im Frühjahr 1667 standen 30000 osmanische

30 Vgl. neben den oben genannten Abhandlungen Eickhoff, Venedig, Wien und die Osmanen (wie Anm. 2), S. 27-32; Setton, Venice, Austria, and the Turks (wie Anm. 2), S. 110-113; HammerPurgstall, Geschichte des Osmanischen Reiches (wie Anm. 6), Bd 3, S. 257-260, auch Kretschmayr, Geschichte von Venedig (wie Anm. 11), S. 313-317 und S. 333-335; Jorga, Geschichte des Osmanischen Reiches (wie Anm. 11), S. 122-134; Petacco, L'ultima crociata (wie Anm. 3), S. $123-130$.

$31 \mathrm{Zu}$ diesen Unternehmungen vgl. Eickhoff, Venedig, Wien und die Osmanen (wie Anm. 2), S. 49-55; Luigi Rangoni Machiavelli, Giovanni Fiomano Bichi Balí Gran Priore di Capua (1613-1676) e la battaglia dei Dardanelli del 1657. In: Rivista del Sovrano Militare Ordine di Malta, 3 (1939), 4, S. 3-6. Zu den im Kontext der Belagerung von Kreta durchgeführten maritimen Operationen vgl. ausführlich Anderson, Naval wars in the Levant (wie Anm. 3), und verschiedene Monografien zur Geschichte Venedigs und des Osmanischen Reiches.

32 Zum damaligen militärischen Potenzial des Osmanischen Reiches vgl. einführend Gábor Ágoston, The Most Powerful Empire: Ottoman Flexibility and Military Might. In: Empires and Superpowers: Their Rise and Fall. Ed. by George Zimmar [u.a.], Washington, DC 2005, S. 127-171. 33 Zur bedeutenden Rolle von Ahmet Köprülü vgl. stellvertretend Eickhoff, Venedig, Wien und die Osmanen (wie Anm. 2), S. 170-175. 
Truppen zum Angriff auf Kandia bereit. ${ }^{34}$ Der Befehl über die Flotte lag in den Händen des frisch ernannten Großadmirals Silahdar Yusuf Pascha. ${ }^{35}$

Die venezianischen Truppen und Kontingente der ausländischen Hilfstruppen standen unter dem Oberkommando von »Capitano Generale« Francesco Morosini (der 1688 zum Dogen gewählt werden sollte), assistiert von dem Piemontesen Marchese Geronimo Francesco Villa und dem Deutschen Georg Friedrich Graf von Sparr. ${ }^{36}$ Als »Proveditore Generale« und Kommandant von Kandia amtierte der Venezianer Antonio Barbaro, der bald durch Bernardo Nani ersetzt werden sollte. ${ }^{37}$

Für Morosini stellte sich die Lage im Frühjahr 1667 als wenig optimistisch dar. Seine ohnehin nicht umfangreichen Verbände waren im vorhergehenden Winter durch Epidemien und Hunger weiter reduziert worden. Für den Moment blieb nur die Option, auf das Eintreffen von frischen Entsatztruppen zu warten. ${ }^{38}$ Die Situation verschlechterte sich weiter, als es den Osmanen gelang, Geschützbatterien in der Nähe des Hafens von Kandia zu platzieren und eine geregelte Versorgung der Belagerten auf dem Seeweg zu verhindern. Diese konnte daher nur noch unter großen Gefahren und in unregelmäßigen Abständen nachts durchgeführt werden. Am 28. Mai 1667 begannen die osmanischen Angriffe auf Kandia. Deren ausführliche Schilderung kann im Rahmen dieses Beitrags jedoch nicht geleistet werden. Ungeachtet der numerischen Überlegenheit, und des massiven Einsatzes von Mineuren zur Unterhöhlung der Festungsanlagen gelang es den Angreifern jedoch bis zum September nur einige Vorwerke zu erobern. ${ }^{39}$

Heftige Regenfälle, der Ausbruch von Epidemien im osmanischen Lager und die sich durch die Herbststürme verschlechternde logistische Versorgungssituation der Angreifer ließen Großwesir Köprülü die Angriffe abbrechen und einen neuen Versuch der Eroberung auf das nächste Frühjahr verschieben.

$34 \mathrm{Zu}$ den letzten drei Jahren des Krieges um Kandia vgl. Candiani, Francia, Papato e Venezia (wie Anm. 2), S. 829-887; Eberhard Werner Happel, Historia Moderna Europae, Ulm 1692, S. 865-867; Staatsgeschichte der Republik Venedig, 3. T., Riga 1777, S. 565-570; Setton, Venice, Austria, and the Turks (wie Anm. 2), S. 193-243; Anderson, Naval Wars in the Levant (wie Anm. 3), S. 172-177.

35 Zur Karriere von Silahdar Yusuf vgl. ausführlich Thomas Higgons, The History of Jussuf Bassa, Captain General of the Ottoman Army at the Invasion of Candia, London 1684.

36 Vgl. den zeitgenössischen Bericht von François Savinien d'Alquié, Memoires du voyage de M. de Ville ou Histoire du siege de Candie, Paris 1670.

37 Der »Proveditore« Bernardo Nani sollte noch im Sommer $1668 \mathrm{im} \mathrm{Kampf} \mathrm{fallen.}$

38 Zur Rolle der Entsatztruppen, speziell der bayerischen Kontingente, vgl. Geschichte des kurbayerischen Heers (wie Anm. 5), S. 255f.

39 Vgl. den Augenzeugenbericht des Ingenieurs Johann Bernhard Scheither, Novissima praxis militaris, oder neu-vermehrte und verstärckte Festungs-Bau- und Kriegs-Schule, Braunschweig 1672; Bigge, Der Kampf um Candia (wie Anm. 12), S. 124-127. 
Im Winter 1667/68 erfolgten in Venedig und am päpstlichen Hof intensive Überlegungen und Verhandlungen über die Stärkung der Verteidiger gegen den bevorstehenden Angriff. Erneute Appelle an die europäischen Fürstenhöfe um Unterstützung trugen insofern Früchte, als Herzog Friedrich von Braunschweig-Lüneburg die Entsendung von drei Regimentern, der bayerische Kurfürst Ferdinand Maria ein Spezialcorps, der Herzog von Savoyen 500 Söldner, die Erzbischöfe von Mainz und Köln und die Bischöfe von Straßburg und Paderborn Einheiten von jeweils 400 Mann versprachen. Auch die Kurie kündigte die Entsendung von 400 Söldnern unter dem Kommando von General Muzio Mattei an und gestattete der Serenissima die Anwerbung von 700 Söldnern im Kirchenstaat. Der Malteserorden bereitete wie in den Jahren zuvor die Entsendung seiner Galeerenflotte nach Kreta vor. Diesmal befanden sich 63 Ordensritter und 350 Söldner unter dem Oberbefehl von Ettore de Fay la Tour an Bord..$^{40}$ Obgleich auch Kaiser Leopold I. die Aufstellung eines Kontingents von 2000 Mann Infanterie für den Entsatz von Kandia anordnete, sollten logistische Verzögerungen einen Kampfeinsatz schließlich verhindern. ${ }^{41}$

Papst Klemens IX. propagierte den Kampf um Kandia als Heiligen Krieg und steuerte weitere 1800000 Scudí als Unterstützung bei. Zum Entsatzplan gehörte des Weiteren die Zusammenarbeit einer aus venezianischen, maltesischen und päpstlichen Kriegsschiffen bestehenden Flotte, welche die Versorgungsrouten der Osmanen unterbrechen bzw. blockieren sollte. Diese Flotte sollte später von einem Verband aus neun spanischen Kriegsschiffen unterstützt werden. Der Vizekönig von Sizilien war von der Regierung in Madrid angewiesen worden, diese in den Häfen von Messina und Augusta instand zu setzen. Auch hier verhinderten logistische Probleme einen Einsatz in der Kampagne des Jahres $1668 .^{42}$

Die Verhandlungen mit Frankreich bezüglich militärischer und maritimer Hilfe gestalteten sich kompliziert. Ludwig XIV. wollte die traditionell guten Handelsbeziehungen zwischen Frankreich und dem Osmanischen Reich nicht nachhaltig stören, spielte doch gerade der Handel mit der Levante nach wie vor eine bedeutende Rolle für Frankreichs See- und Handelszentren Marseille, Toulon oder auch Lyon. Die unter Colbert neu gegründete »Compagnie du Levant« dokumentiert diese Bedeutung. Der französische König zögerte daher mit seinem Einverständnis für die offizielle Entsendung von Truppen in das östliche Mittelmeer; er erlaubte Venedig lediglich die Aushebung von Truppen auf französi-

40 Dal Pozzo, Historia della Sacra Religione (wie Anm. 7), vol. 2, S. 354f.; Rossi, Storia della Marina (wie Anm. 27), S. 70f.

41 Bigge, Der Kampf um Candia (wie Anm. 12), S. $125 f$.

42 Vgl. ausführlich zur Kampagne von 1668: Der Neu-eröffneten Pforten, Fortsetzung oder continuierter Bericht der Türkischen Monarchie Staats-Maximen, Augsburg 1701, S. 95-140. 
schem Territorium. Die vom Duc de la Feuillade schließlich zusammengestellten Korps durften nicht auf französischen Schiffen nach Kandia gebracht werden. ${ }^{43}$ Sie segelten schließlich unter der Flagge des Malteserordens. Frankreich erklärte sich vorerst nur zur Beisteuerung von 400000 Scudí für die christlichen Kriegskosten bereit.

Auch im Winter 1667/68 war eine solide unitas christiana eine Illusion: Die neue osmanische Kampagne wurde durch die Lieferung von Kriegsgerät, Pulver und Eisenwaren durch englische und niederländische Schiffe unterstützt. ${ }^{44}$

Mit oben aufgelisteter Unterstützung belief sich die Stärke der Verteidiger von Kandia im Frühjahr 1668 auf etwa 5000 Infanteristen, Kanoniere, Pioniere und andere Spezialtruppen. Hinter der Kampfstärke und Kampfmoral dieser häufig sehr schnell und unter zweifelhaften Methoden angeworbenen Entsatztruppen standen jedoch Fragezeichen. Insgesamt verfügten die Verteidiger über 90 großkalibrige und 300 leichtere Geschütze. ${ }^{45}$ Die Angreifer waren klar in der Überzahl, vor allem als im Frühjahr 1668 weitere 3500 Janitscharen, 4000 ägyptische und syrische Soldaten, 1000 armenische Mineure und Artilleristen sowie 1000 Sipahi auf Kreta angelandet wurden. ${ }^{46}$

Im August hatten die osmanische Artillerie und die Mineure verschiedene Bastionen und Mauern der Festung stark beschädigt und Breschen zum Angriff für Sturmtruppen vorbereitet. ${ }^{47}$ Ein schon geplanter Angriff wurde infolge der Nachrichten frisch eingetroffener Entsatztruppen und des Auftauchens der venezianischen, maltesischen, päpstlichen und florentinischen Flottenverbände noch einmal verschoben. Dabei handelte es sich bei diesen Entsatztruppen um 2500 Söldner aus Venedig, Hannover und Braunschweig unter dem Befehl von Catterino Cornaro. ${ }^{48}$ Mit der venezianischen Flotte war auch Francesco Morosini eingetroffen und übernahm von nun an erneut das Oberkommando vor Ort. Seine Inspektion der Festung, der Artillerie und der verfügbaren Truppen stimmte wenig optimistisch, längere Zeit aushalten zu können. Erneut wurden Boten nach

43 Vgl. Anonym, Journal de l'expedition de M. de la Feuillade pour le secours de Candie, Lyon 1669.

44 Bigge, Der Kampf um Candia (wie Anm. 12), S. 154.

45 Detaillierte Augenzeugenberichte stammen von dem bereits erwähnten Buch des Militäringenieurs Johann Bernhard Scheither, Novissima praxis militaris (wie Anm. 39), Kap. 3; vgl. auch die Augenzeugenberichte in: Das lange bestrittene Königreich Candia (wie Anm. 5).

46 Zur Kampagne des Jahres 1668 vgl. Bigge, Der Kampf um Candia (wie Anm. 12), S. 153-170; Roger Palmer of Castlemaine und Johann Heinrich Bodmer, Das von den Türken bedrängte Candia, Frankfurt a.M. 1669.

47 Bigge, Der Kampf um Candia (wie Anm. 12), S. 155.

48 Ebd., S. 157. 
Venedig, Palermo, Neapel und Rom mit der Bitte entsandt, weitere Verstärkung und modernes Kriegsgerät zu schicken.

Gleichzeitig entwickelte man einen neuen Plan zur Unterstützung der Verteidiger und der christlichen Sache im zentralen und östlichen Mittelmeer. Die intensive Auseinandersetzung mit einem auch propagandistisch und strategisch umfassender vorbereiteten christlichen Gegenschlag war eng mit der Person des erst wenige Monate zuvor am 20. Juni 1667 in das höchste kirchliche Amt gelangten Giulio Rospigliosi alias Klemens IX. verknüpft.

Mehr noch als sein Vorgänger Alexander VII. identifizierte sich der neue Papst mit der alten Idee eines christlichen Kreuzzugs. Es ist in diesem Kontext kein Zufall, dass Sommer und Herbst 1667 einen »medialen« Höhepunkt im Leben der berühmten Konvertiten Padre Ottomano und Baldassare Diego Loyola darstellten. Nicht zuletzt auf Betreiben der Kurie erfolgte die Publikation zahlreicher Sonette, Gedichte und mit Porträts versehener Pamphlete über die lobenswerten Aktivitäten und das gottgefällige Leben der beiden; vielleicht bekanntestes war der in Ferrara publizierte »Lode del P. Ottomano ${ }^{49}$

Gleichzeitig mit dieser Dynamisierung der katholischen Propaganda kam es zu Überlegungen, Padre Ottomano direkt in den theatrum belli einzubinden. Der berühmte Dominikaner lebte damals - wie schon erwähnt - im Konvent von SS. Annunziata in Paris und hatte bereits im Jahr zuvor drei Briefe des Generalvikars der Griechisch-Orthodoxen Kirche von Alexandria erhalten, in denen er gebeten wurde, sich in den Osten zu begeben und einen Aufstand der griechischen und armenischen Christen gegen die osmanischen Besatzer zu unterstützen. Den Worten des Generalvikars gemäß würden die Aufständischen Padre Ottomano als ihren »König « anerkennen. ${ }^{50}$ Dies sei auch der sehnliche Wunsch des Patriarchen von Alexandria, des Fürsten der Wallachai, des russischen Zaren und der Oberen der Christen in Äthiopien. Im Falle einer Einigung könne das Osmanische Reich von verschiedenen Flanken angegriffen werden.

Wenige Wochen später traf in Paris tatsächlich ein Schreiben des Wallachenprinzen Gregor Basil Rákóczi, eines Sohnes des 1660 verstorbenen Fürsten über Siebenbürgen, Georg II. Rákóczi, ein, das ähnliche Vorschläge enthielt. ${ }^{51}$ Moderne Historiker stellen die dem Padre angedachte Rolle in den Kontext des armenischen Unabhängigkeitskampfes. ${ }^{52}$

49 Anonym, Lode del P. Ottomano, Ferrara 1667.

50 Hier wird die zweite Auflage (1698) von Bulgarinis Werk zitiert; vgl. Bulgarini, Vita del Padre (wie Anm. 10), S. 511-513.

51 Bulgarini, Vita del Padre (wie Anm. 10), S. 513.

52 Louis Nalbandian, The Armenian Revolutionary Movement, Berkely, Los Angeles, CA, London 1963, S. 20-22. 
In der Kurie wurden damals Pläne entwickelt, wie die Beteiligung des Padre Ottomano am Kampf um Kandia und ein Angriff der vereinigten Flotten von Venedig, Malta, Florenz, dem Kirchenstaat und anderen Verbänden auf die Dardanellen propagandistisch genutzt werden könnte. Das Szenario solch eines umfassenden Volksaufstandes gegen die Osmanen und eines flankierenden maritimen Unternehmens gegen vitale osmanische Seerouten und Handelsstützpunkte musste für die Hauptprofiteure des Levante-Handels, Frankreich, England oder die Niederlande, als massive Bedrohung ihrer Handelsinteressen aufgefasst werden. Bei Erfolg dieser Pläne war eine politische Erschütterung und Destabilisierung der gesamten Region des östlichen Mittelmeers zu erwarten.

Die verfügbaren Archivalien geben keine Hinweise, ob es Padre Ottomano selbst war, der ausländische Diplomaten am französischen Hof über diese Pläne informierte; dokumentiert ist, dass der venezianische Gesandte am Hof Ludwigs XIV., Marco Antonio Giustiniani, umgehend den Senat der Serenissima über diese Entwicklungen in Kenntnis setzte. Damit war ein wirksamer Entsatz für das belagerte Kreta $\mathrm{zu}$ erwarten. Im Sommer 1667 fanden geheime Verhandlungen zwischen der Kurie, dem General der Dominikaner, Giovanni Battista de Marinis, und Vertretern Venedigs über die Einsatzmöglichkeiten des berühmten Padre statt. Die Venezianer erreichten schließlich den gemeinsamen Entschluss der Verhandlungspartner, dass Padre Ottomano zuerst nach Kandia reisen und dort die Auflösung der Moral der osmanischen Truppen vorantreiben solle..$^{53}$

\section{Von Paris nach Rom}

Am 27. Juli 1667 verließen Padre Ottomano und sein Sekretär Padre Ignozzi di Terracusa Paris mit dem Ziel Venedig. Nach ihrer Ankunft trafen sie sich mit dem Dogen Domenico II. Contarini und dem Senat, mit denen sie darüber diskutierten, wie ein rascher Entsatz Kandias am effektivsten zu erreichen sei. Es wurde schließlich beschlossen, dass Padre Ottomano mit dem Hauptverband frischer venezianischer Truppen nach Kreta aufbrechen solle. Dies musste anschließend von dem Dominikaner-General und Papst Klemens IX. bestätigt und genehmigt werden.

Es bleibt anzumerken, dass bis zu diesem Zeitpunkt keinerlei Indizien für eine aktive Beteiligung, geschweige denn Initiative des Padre an oben skizzierten

53 Vgl. Vincenzo Abbondanza, Dizionario storico delle vite di tutti monarchi ottomani, Rom 1786, S. 81-83; Le Grand Dictionnaire historique. Ed. par Louis Moreri, t. 3, Lyon 1674, S. 126; Antonio Arrighi, De vita et rebus gestis Francisci Mauroceni, Padua 1749, S. 153-155, und die neuere Studie von Candiani, Francia, Papato e Venezia (wie Anm. 2), S. 829-887. 
Plänen vorlagen. Der Dominikaner behielt seinen zurückgezogenen Lebensstil und seine passive Attitüde gegenüber seinem Umfeld bei. Weder in seiner Zeit in Paris noch während seines neuen Aufenthalts in Venedig gibt es Hinweise auf die Teilnahme an politischen Zirkeln oder den Besuch der intellektuellen Salons. ${ }^{54}$ Zeitgenössische bzw. eine Generation später schreibende Biografen wie Ottaviano Bulgarini, Jacques de Jant, Felice Tempia u.a. schildern sein Verhalten als zurückhaltend und bescheiden (»tutt'umile e modesto«). ${ }^{55} \mathrm{Ob}$ diese Beschreibungen seiner sozialen Rolle als Mönch entsprechen sollten oder als Hinweise auf einen lethargischen und passiven Charakter interpretiert werden können, ist im Nachhinein schwer zu ermitteln.

Glauben wir den Biografen, dann änderte sich das Verhalten des Padre Ottomano nach seinem Besuch von Venedig im Spätsommer 1667 drastisch. Die Gesamtschau seiner Vita legt die Vermutung nahe, dass seine nun beginnende Rolle als uomo politico das Werk einer Gruppe von Einflüsterern und Tutoren aus dem Umkreis der Römischen Kurie und dem venezianischen Senat war. Noch am 1. November 1664 hatte der französische chargé d'affaires am Hof von Turin, Baron Servien, nach einer längeren Konversation mit dem Padre nach Paris gemeldet, dieser habe keinerlei Interesse, für sich fürstliche Rechte und Prätentionen in Anspruch zu nehmen. Sein Ziel sei es, ein von geistlichen Exerzitien geprägtes Leben und einen mönchischen Lebensstil zu führen. ${ }^{56}$ Wir können daher mit an Sicherheit grenzender Wahrscheinlichkeit annehmen, dass der ambitionierte «quadro d'un piano politico antiturco «57 nicht auf den Padre Ottomano zurückgeht. Der diesen Plänen - nicht zuletzt aufgrund der oben genannten wirtschaftlichen und politischen Gründe - skeptisch gegenüberstehende französische Hof sah damals ausschließlich die Römische Kurie und die Venezianer als Urheber und Betreiber. ${ }^{58}$ Gemäß einigen Zeitzeugen war der venezianische chargé d'affaires am Heiligen Stuhl, Antonio Grimani, die zentrale

54 Vgl. auch Bulgarini, Vita del Padre (wie Anm. 10), S. 500-508; Youssuf Missak Effendi, Le Père Ottoman. In: Revue d'Histoire Diplomatique, (1903), S. 350-378, hier S. 365-367.

55 Vgl. die Zitate der Zeitgenossen, abgedruckt in Missak Effendi, Le Père Ottoman (wie Anm. 54), S. 365-367.

56 Vgl. Baron Serviens Brief an Louis XIV. vom 1.11.1664, Archives Nationales de France, Paris (ANF), Affaires etrangères, Savoie, correspondence xlviii, f. 138, hier zit. nach Missak Effendi, Le Père Ottoman (wie Anm. 54), S. 365.

57 Bono, Schiavi musulmani (wie Anm. 22), S. 301.

58 Vgl. das Schreiben von Niccolò Pietro Bargellini, des apostolischen Nuntius in Paris, an Kardinal Rospigliosi vom 5. und 19.7.1669, Archivio Segreto Vaticano (ASV), Nunziatura di Francia, CXXXVII, f. 692, 705; hier zit. nach Callus, Il P. Domenico Ottomano (wie Anm. 10), S. $108 f$. 
Figur, die den Papst von der Bedeutung einer Teilnahme des Padre Ottomano an der zukünftigen Kampagne zum Entsatz Kretas überzeugte. ${ }^{59}$

Die überlieferte Korrespondenz dokumentiert, dass der Padre und sein Umfeld den französischen Hof nicht über die Hintergründe seiner Abreise aus Paris informierten. Dies hing sicherlich mit der zu erwartenden ablehnenden Haltung der französischen Regierung gegenüber einer Rebellion auf dem griechischen Archipel und einer Destabilisierung des östlichen Mittelmeers und des Nahen Ostens zusammen.

Für die politische Öffentlichkeit und europäischen Regierungen ergab sich ein klareres Bild, als der Padre Ottomano im Januar 1668 von Venedig nach Rom reiste und dort mit verschiedenen Kardinälen und auch dem Papst zusammentraf. In den Kreisen der Kurie herrschte eine erwartungsvolle, optimistische Stimmung; vieles sprach für einen Erfolg der Mission des Padre Ottomano: Sein jüngerer »Bruder«, Sultan Mehmet IV., sei beim Großteil der osmanischen Bevölkerung unbeliebt, der seit über zwanzig Jahren andauernde Krieg um Kandia würde früher oder später zum finanziellen Zusammenbruch des Osmanischen Reiches führen. ${ }^{60}$ Durch die sich im Verlauf des 17. Jahrhunderts am osmanischen Hof durchsetzende Regel des Seniorats, also der Nachfolge des ältesten männlichen Mitglieds der Dynastie als Sultan, sei die Position des Padre Ottomano als Prätendent weiter gestärkt worden. Dieses älteste Mitglied der Dynastie musste keineswegs der Sohn des Vorgängers sein.

Der Dominikaner selbst ließ verlauten, er hoffe, so bald wie möglich in das östliche Mittelmeer reisen zu können. Seine Präsenz würde sicherlich die osmanischen Truppen und auch die griechische Bevölkerung zur Rebellion gegen die osmanische Führung bewegen. Durch koordiniertes Agieren mit den griechischen Patriarchen ließen sich die griechische Bevölkerung und die "guten« Osmanen bestimmt auf die Seite des Padre ziehen. ${ }^{61}$ Primäres Ziel des Aufstands war eine entscheidende Schwächung Mehmets IV. Perspektivisch sollte ein - wenn möglich sich bis auf die Levante erstreckendes - christliches Reich im östlichen

59 Bigge, Der Kampf um Candia (wie Anm. 12), S. 136. Zur Rolle des Padre Ottomano in Kandia und auf Zakyntos vgl. die Hinweise in Samuele Romanin, Storia documentata di Venezia, 10 vol., Venedig 1912-1921, hier vol. 7; d'Alquié, Les Mémoires (wie Anm. 5), t. 1, S. 6f.; Petacco, L'ultima crociata (wie Anm. 3), S. 123-125; Hammer-Purgstall, Geschichte des Osmanischen Reiches (wie Anm. 6), Bd 6, S. 238; Eickhoff, Venedig, Wien und die Osmanen (wie Anm. 2), S. 231.

60 Schreiben des venezianischen Botschafters bei der Römischen Kurie, Antonio Grimani, vom 14.4.1668, hier zitiert nach Missak Effendi, Le Père Ottoman (wie Anm. 54), S. 372f.

61 Ebd.; vgl. auch das Schreiben von Marc'Antonio Giustiniani an Padre Ottomano vom 10.5.1668, hier zit. nach Bulgarini, Vita del Padre (wie Anm. 10), S. 533. 
Mittelmeer errichtet werden. ${ }^{62}$ Inwieweit es gelingen konnte, die durch ihren Status als »dhimmi« (Beschützte) relativ gut behandelten christlichen Untertanen auf dem Balkan zu einer Revolte zu veranlassen, kann im Rahmen dieses kurzen Beitrags nicht näher untersucht werden. ${ }^{63}$

Aus diesen weitgespannten Plänen spricht insgesamt ein überraschendes Maß an Optimismus, um nicht zu sagen Naivität. ${ }^{64}$ Mangels Alternativen erschien es vielen Beteiligten als letzte Option, die Situation im östlichen Mittelmeer zum Positiven zu wenden. Auch nachdem der Padre Ottomano im Mai 1668 Rom verlassen hatte, dokumentiert die Korrespondenz des Papstes die Hoffnungen der Kurie auf einen durch den Dominikaner provozierten Aufstand gegen Mehmet IV. in Griechenland und dem südlichen Balkan. ${ }^{65}$ In der Zwischenzeit war Francesco Morosini über die bevorstehende Mission des Padre Ottomano umfassend informiert worden; mit Blick auf den Entsatz von Kandia war es ihm überlassen, auf welche Weise der angebliche ältere Bruder des Sultans dort am effektivsten eingesetzt werden sollte. ${ }^{66}$

62 Vgl. Bulgarini, Vita del Padre (wie Anm. 10), S. 544; vgl. auch das Schreiben Antonio Grimanis an den Gouverneur von Souda, Bernardo Nani, vom 15.5.1668, abgedruckt in de Daru, Geschichte der Republik Venedig (wie Anm. 12), Bd 5.

63 In den letzen Jahren erschienen verschiedene Untersuchungen, die sich mit der komplexen Problematik der religiösen und sozialen Identität der Bevölkerung auf dem Balkan und dem Konvertitenwesen im Osmanischen Reich beschäftigen. Vgl. Anton Minkov, Conversion to Islam in the Balkans: Kisve Bahas petitions and Ottoman Social Life, 1670-1730, Leiden 2004; Marc David Baer, Honoured by the Glory of Islam: Conversion and Conquest in Ottoman Europe, Oxford 2008; Tijana Krstić, Illuminated by the Light of Islam and the Glory of the Ottoman Sultanate Self-Narratives of Conversion to Islam in the Age of Confessionalization. In: Comparative Studies in Society and History, 51 (2009), 1, S. 35-63; Tijana Krstić, Contested Conversion to Islam. Narratives of Religious Change in the Early Modern Ottoman Empire, Palo Alto 2011; Conversion and Converts to Islam in Ottoman Historiography of the Fifteenth and Sixteenth Centuries. In: Writing History at the Ottoman Court - Editing the Past, Fashioning the Future. Ed. by E. Cipa and E. Fetvaci, IN 2013, S. 58-79.

64 Der italienische Historiker Salvatore Bono kommentiert »l'ingenua ristrettezza mentale con la quale Roma volute le reazioni di un avversario guidato da un'ideologia e da concezioni politiche affatto opposte; agli occhi dei turchi appariva uno scherzo grottesco la richiesta di cingere con la spade del Profeta un turco fattosi prete cattolico«. Bono, Schiavi musulmani (wie Anm. 22), S. 301f. Ähnliche Kommentare über die Naivität des Plans finden sich auch bei Eickhoff, Venedig, Wien und die Osmanen (wie Anm. 2), S. 244-246; Setton, Venice, Austria, and the Turks (wie Anm. 2), S. 220f.

65 Hier zit. nach Missak Effendi, Le Père Ottoman (wie Anm. 54), S. 374; Bigge, Der Kampf um Candia (wie Anm. 12), S. 136.

66 Vgl. Bigge, Der Kampf um Candia (wie Anm. 12), S. 136; Missak Effendi, Le Père Ottoman (wie Anm. 54), S. 374, und das semi-fiktive Werk von A. von Tromlitz (= Karl August Friedrich von Witzleben), Die Belagerung von Candia, Bd 1, Dresden, Leipzig 1832, S. 66-68. 
Diese Entwicklungen in Italien - obwohl offiziell noch geheim - stießen nicht nur in den politischen Kreisen der katholischen Welt auf großes Interesse. In welch hohem Maß auch die britischen Handelsherren und Konsortien, namentlich die Vertreter der "Levant Company«, von den Vorgängen in Rom und Venedig beunruhigt wurden, zeigt ein damals in London in lateinischer Sprache publiziertes Buch (»Historia de tribus hujus saeculi impostoribus «), ${ }^{67}$ das den Dominikaner-Pater als Betrüger zu entlarven suchte. Als Verfasser des Werks gilt der bekannte Autor und Jurist John Evelyn. Das unschwer als Auftragsarbeit zu identifizierende Buch fand umgehend großes Interesse auf dem Buchmarkt und erschien noch im gleichen Jahr in englischer und deutscher Übersetzung. ${ }^{68}$

Mitte Mai 1668 war Padre Ottomano in Civitavecchia, dem Heimathafen der päpstlichen Flotte, eingetroffen. Offensichtlich war der frühere Plan aufgegeben worden, den berühmten Passagier auf venezianischen Kriegsschiffen nach Kreta $\mathrm{zu}$ transportieren. In Civitavecchia bereitete sich im Mai eine aus fünf Galeeren, zwei Transportschiffen und einer felucca bestehende päpstliche Flotte auf die Passage nach Kreta vor. Zuerst sollte Padre Ottomano auf dem Flaggschiff der Flotte, der capitana von Admiral Balí Vincenzo Rospigliosi, fahren. ${ }^{69}$ Als die Flotte am 19. Mai schließlich den Hafen verließ, befand sich der Padre allerdings auf der Galeere San Pietro. Sie wurde von einem Bekannten aus seiner Zeit auf Malta, Komtur Buontempi, kommandiert. ${ }^{70}$

67 Der volle Titel der englischen Ausgabe lautet: The history of the three late, famous impostors, viz. Padre Ottomano, Mahomed Bei and Sabatai Sevi the one, pretended son and heir to the late Grand Signior, the other, a prince of the Ottoman family, but in truth, a Valachian counterfeit, and the last, the suppos'd Messiah of the Jews, in the year of the true Messiah, 1666: with a brief account of the ground and occasion of the present war between the Turk and the Venetian: together with the cause of the final extirpation, destruction and exile of the Jews out of the Empire of Persia, London 1667.

68 Historia de tribus hujus saeculi impostoribus. Das ist die Beschreibung des P. Ottomano, Mahommed Bei und Sabati Sevi. Aus dem Engl. übers., Frankfurt a.M. 1668. Das Thema wurde aufgegriffen in Omniana. Ed. by Robert Southey and Samuel Taylor Coleridge, vol. 1, London 1812, S. 60-69, und von dem anonymen Autor der Celebrated Claimants from Perkin Warbeck to Arthur Orton, London 1874, S. 42-45.

69 Vgl. den Brief Balí Vincenzo Rospigliosis an Luogetenente Banchieri vom 11.5.1668, hier zit. nach Bulgarini, Vita del Padre (wie Anm. 10), S. 536f.; vgl. auch Callus, Il P. Domenico Ottomano (wie Anm. 10), S. 95.

70 Bulgarini, Vita del Padre (wie Anm. 10), S. 537. Über das Kriegsschiff, auf dem der Padre Ottomano in das östliche Mittelmeer fuhr, gibt es verschiedene, sich widersprechende Angaben. Gemäß Oberst Wilhelm Bigge - Bigge, Der Kampf um Candia (wie Anm. 12), S. 136 - segelte er auf dem Flaggschiff, Rospigliosis capitana. Dem wird in der »Relatione del viaggio fatto in Levante dall’Eccmo. Sign. Balí Frà Vincenzo Rospigliosi, generalissimo delle galere della Santità di N. S. Papa Clement IX l'anno 1668« widersprochen - aufbewahrt im Archivio Rospigliosi in Rom, 


\section{Der Padre Ottomano auf Kreta}

Von Civitavecchia segelte die päpstliche Flotte in südlicher Richtung. Nach einem kurzen Aufenthalt im Hafen von Neapel zur weiteren Verproviantierung ging es weiter nach Messina, wo sich die päpstliche Flotte mit dem Galeerenverband des Malteserordens vereinigen sollte. Widrige Winde verhinderten eine zügige Fahrt. Es dauerte daher bis zum 5. Juni, bis die angestrebte Zusammenführung der Flottenverbände in Messina vollzogen wurde. Zusammen mit sechs maltesischen Galeeren unter dem Kommando von Generalkapitän Clemente Accarigi wurde Kurs auf das Kap von S. Maria an der apulischen Küste genommen. ${ }^{71}$ Von dort kreuzten die Schiffe über die Adria nach Korfu, wo man am 15. Juni eintraf und Admiral Rospigliosi Briefe von Francesco Morosini vorfand, die zu einer raschen Weiterfahrt zum Kap Spada drängten, um sich mit der vor der Insel Koite (in den ital. Dokumenten »San Teodoro«) ankernden venezianischen Flotte zu vereinigen. ${ }^{72}$ Deren Stärke wird mit 5 Galeassen, 15 Galeeren und einer Anzahl von Galleotten und Brigantinen angegeben. Kurz nach dem Eintreffen der päpstlichmaltesischen Flotte erreichte ein von drei Galeeren geschützter Verband von neun venezianischen Transportschiffen mit Truppen und Kriegsgerät Korfu. Zusammen mit diesen setzten die päpstlichen und maltesischen Schiffe am 25. Juni ihren Kurs nach Kap Spada und Kreta fort. Die Besatzungen von Handelsschiffen von Ragusa (heute Dubrovnik) berichteten, dass an der Küste von Kythira (ital. Cerigo) zehn Korsarenschiffe auf Beute warteten. Admiral Rospigliosi schätzte seine Flotte als stark genug ein, eventuellen Angriffen standzuhalten, und gab Befehl zur Weiterfahrt.

Er sollte recht behalten; die Korsaren wagten keinen Angriff auf die 14 maltesisch-päpstlich-venezianischen Galeeren und die anderen Schiffe des Verbandes. Am 7. Juli erreichte man Kap Spada, wo wie vereinbart die venezianische Flotte wartete. Zusammen segelte man zur Insel Koite. Am 18. Juli kam es auf Morosinis capitana zu einem Treffen mit dem venezianischen Befehlshaber selbst, mit Admiral Rospigliosi, Generalkapitän Accarigi und weiteren hochrangigen Offizieren, um einen Einsatzplan für Padre Ottomano zur Provokation einer Rebellion unter den osmanischen Truppen zu entwickeln. ${ }^{73}$

hier zit. nach Callus, Il P. Domenico Ottomano (wie Anm. 10), S. 187. Gemäß der »Relatione« reiste er auf der Galeere San Caterina.

71 Vgl. Bigge, Der Kampf um Candia (wie Anm. 12), S. 137; Fontana, L'Origine della Sacra et eminentissima Religione (wie Anm. 9), S. 340; zu den Unternehmungen Accarigis im Jahr 1668 vgl. auch Brusoni, Historia dell'ultima Guerra (wie Anm. 6), S. 213-215.

72 Bigge, Der Kampf um Candia (wie Anm. 12), S. 138.

73 Ebd., S. 151-153. 
Am nächsten Tag landete der Padre zusammen mit den Entsatztruppen bei Kandia. ${ }^{74}$ Er wurde umgehend von Gouverneur Bernardo Nani in der Festung von Souda auf der Insel Agio Nikolaos empfangen. Noch am selben Tag wurde beschlossen, Briefe an Großwesir Köprülü, Großadmiral Silahdar Yusuf Pascha und andere hochrangige osmanischen Offiziere $\mathrm{zu}$ senden, in denen sich der Padre als ältester Bruder von Sultan Mehmet IV. vorstellte.

Den osmanischen Offizieren wurden im Fall eines Rebellierens gegen den Sultan hohe Belohnungen versprochen. ${ }^{75}$ Zum Überbringen der Post dienten osmanische Kriegsgefangene und Sklaven. Erster Adressat war Großadmiral Silahdar Yusuf Pascha, der jedoch - entgegen den Hoffnungen der Christen - den Überbringer, den Quellen zufolge ein gewisser Yusuf, sofort in Einzelhaft nahm und das Schreiben - angeblich ungelesen - umgehend an den Großwesir weiterschickte. Zusätzlich erfolgte das strikte Verbot für alle Offiziere und Mannschaften, Briefe und Nachrichten von der christlichen Seite zu empfangen.

Ob Großwesir Ahmet Köprülü die ihm zugetragenen Schreiben studierte, ist nicht überliefert. Sollte er dies getan haben, hinterließ er keine erkennbaren Spuren, sandte er sie doch versiegelt an Sultan Mehmet IV. nach Istanbul. ${ }^{76}$ Insgesamt bleibt - soweit es aus heutiger Sicht die Quellen gestatten - festzuhalten, dass diese mit »Osman« unterzeichneten Schreiben keinen tieferen Eindruck auf der Seite des Feindes machten, geschweige denn Truppenteile zum Aufstand oder zum Einstellen der Kampfhandlungen veranlassten. Inwieweit es den verantwortlichen Offizieren der osmanischen Truppen gelang, vor den gemeinen Soldaten die Anwesenheit des angeblichen älteren Bruders von Sultan Mehmet IV. auf Kreta zu verbergen, ist im Nachhinein kaum zu ermitteln. Mit Sicherheit wurde dies versucht, war doch eine Reaktion der Truppen auf diese Nachricht unkalkulierbar.

Nach diesem Fehlschlag schlug Morosini andere Methoden vor, die Nachricht von der Gegenwart des berühmten Dominikaners bekannt zu geben und propagandistisch zu nutzen. Es wurde befohlen, mit Armbrustpfeilen Mitteilungen über die Person, Stellung und Absicht des Padre Ottomano in türkischer Sprache in die osmanischen Reihen zu schießen. Damit waren »Anordnungen« des Padre in seiner »Stellung« als erstgeborener Sohn von Sultan Ibrahim an die Soldaten verbunden, dem Großwesir und Sultan den Gehorsam $\mathrm{zu}$ verweigern und $\mathrm{zu}$ desertieren. Auch hier sind wir nicht über die unmittelbaren Effekte dieser Mittei-

74 Zum Aufenthalt des Padre Ottomano auf Kreta vgl. auch Brusoni, Historia dell'ultima Guerra (wie Anm. 6), S. 238-240; Ekkehard Eickhoff, Der Krieg von Kandia in der maritimen Geschichte. In: Saeculum, 21 (1970), S. 373-382.

75 Vgl. Bulgarini, Vita del Padre (wie Anm. 10), S. 548f.

76 Bigge, Der Kampf um Candia (wie Anm. 12), S. 152. 
lungen bei den gewöhnlichen Soldaten informiert. Höchstwahrscheinlich wurden die Schwadronskommandeure (bölük başi) und Regimentskommandeure ( $a \breve{g} a$ ) angewiesen, diese umgehend $\mathrm{zu}$ konfiszieren. Ihre Wirkung wäre ohnehin begrenzt gewesen, da nur ein kleiner Teil der Truppen lesen oder schreiben konnte.

Darüber hinaus hätte sich ein weiteres Problem ergeben: Selbst wenn die Botschaft ihre Adressaten erreicht hätte und diese gelesen worden wäre, wie hätten die osmanischen Truppen wissen können, ob sie auch der Wahrheit entsprach? Auch schien es fraglich, ob sogar die Präsenz des Padre Ottomano auf dem Schlachtfeld bei den gegnerischen Truppen Wirkung zeigen würde. Dabei war zwischen den Truppenteilen zu differenzieren; von den Janitscharen und Sipahis war eine stärkere Loyalität gegenüber Mehmet IV. zu erwarten als von den übrigen Verbänden. Angesichts verschiedener in der venezianischen Marine und auch Infanterie dienender osmanischer Überläufer kann von einem relativ hohen Wissensstand über die Motivation und Verlässlichkeit der auf der Gegenseite dienenden Truppen ausgegangen werden. ${ }^{77}$ Für den Moment - bis bessere Vorschläge entwickelt wurden - musste Padre Ottomano in Wartestellung bleiben.

Obwohl der osmanische Hauptangriff noch nicht begonnen hatte, sahen sich die Belagerten mit verschiedenen ernsten Problemen konfrontiert; eines der dringendsten war die mangelhafte Versorgung mit sauberem Trinkwasser. Im Verlauf des Juli kam es daher zu einer Reihe von osmanisch-christlichen Scharmützeln um Trinkwasser. Der für beide Seiten verlustreichste Kampf um Wasser fand in der Nähe von Calamino statt, wo ein Verband von maltesischen und päpstlichen Söldnern in bereits von Osmanen besetztes Territorium vordrang, um aus Quellen Wasser zu schöpfen. ${ }^{78}$

Innerchristliche Konflikte resultierten aus dem komplexen System der Etikette des Barockzeitalters. Besonders die Malteserritter mit ihrem Anspruch auf eine im Vergleich zu den Truppen aus dem Kirchenstaat, Deutschland, Savoyen und Venedig exklusivere Behandlung provozierten auf diesem Terrain verschiedene Auseinandersetzungen. Generalkapitän Accarigi trug Admiral Rospigliosi diesen Anspruch mehr als einmal vor. Als Letzterer in seiner Eigenschaft als Oberbefehlshaber den Maltesern diese Prärogativen nicht einräumen wollte oder

77 Vgl. E. Natalie Rothman, Conversion and Convergence in the Venetian-Ottoman Borderlands. In: Journal of Medieval and Early Modern Studies, 41 (2011), 3, S. 602-633; Paolo Petta, Stradioti: soldati albanesi in Italia, sec. XV-XIX, Lecce 1996; Domagoj Madunic, Conversion under the Threat of Arms. Converts and Renegades during the War for Crete (1645-1669), Conference Paper für: The Lure of the »Other«: Religious Conversion and Reversion in the Early Modern Mediterranean and Beyond, 4.-5.6.2013, St. Mary's University College, Twickenham, London.

78 Bigge, Der Kampf um Candia (wie Anm. 12), S. 142. 
konnte, weigerten sich die Ordensritter, seinen Befehlen $\mathrm{zu}$ folgen. ${ }^{79}$ Rospigliosis Antwort war diplomatisch: Er könne die Ordensritter nicht zum Bleiben und Kämpfen zwingen, jedoch müssten sie sich für ihre Handlungen vor ihrem Großmeister und dem Papst - seinem Onkel - als geistlichem Oberhaupt der Malteser rechtfertigen. Obgleich Accarigi für den Moment nachgab, blieb die Situation dennoch gespannt und trug nicht zur Stärkung der Moral der christlichen Verteidiger bei.

Ungeachtet dieser inneren Probleme gelang es den Venezianern in einer kombinierten Kampagne von Seestreitkräften und Landtruppen am 3. August, die Insel von Koite zurückzuerobern; Hintergrund dieses riskanten Manövers war es, die dortigen Süßwasserquellen nutzen zu können. ${ }^{80}$

In der Zwischenzeit hatte der Hauptangriff der Osmanen auf Kandia begonnen, und bereits nach relativ kurzer Zeit hatten die osmanische Artillerie und die Mineure eine Bresche in eine dem Hafen zugewandte Bastion geschossen bzw. gesprengt. Das mittlerweile fast täglich einsetzende Bombardement verursachte hohe Verluste in der Festungsstadt. Zu deren Auffüllung sollten Teile der Mannschaften und Marinesoldaten der venezianischen Flotte in die Stadt verlegt werden. ${ }^{81}$ Über die Vorgehensweise, wie dies zu erreichen sei, kam es zu hitzigen Diskussionen zwischen den Offizieren der verschiedenen Kontingente; Generalkapitän Accarigi wollte beispielsweise seine Schiffe nicht dem osmanischen Artilleriefeuer der Landbatterien aussetzen. Admiral Rospigliosi entschied schließlich, dass die auf die Landung vorbereiteten Mannschaften und Marinesoldaten auf den Galeeren nach Kandia zu transportieren seien, während die Segelschiffe, Galeonen, Galeotten und Brigantinen bei Koite vor Anker gehen sollten.

Als die Galeeren am 9. August vor Kandia eintrafen, erwiesen sich die osmanischen Batterien als zu stark und $\mathrm{zu}$ gut platziert, um eine Anlandung der Truppen bei Tag zu riskieren. Erst in der Nacht gelang schließlich die Überführung von 800 Marinesoldaten in die Festungsstadt. ${ }^{82}$ Später wurde bekannt, dass die Präsenz der venezianischen, maltesischen, florentinischen und päpstlichen Galeeren einen für den 10. August geplanten Anlandungsversuch größerer osmanischer Truppenverbände unter dem Befehl des Kapudan-Pascha verhinderte. Die fünfzig osmanischen Transportschiffe änderten ihren Kurs und setzten nach einigen Tagen die Truppen an der Südküste Kretas an Land.

Die im weiteren Verlauf des Augusts in unregelmäßigen Abständen vorgetragenen osmanischen Angriffe brachten keinen entscheidenden Durchbruch. Die

79 Ebd., S. 143.

80 Ebd., S. 146.

81 Ebd., S. 147.

82 Ebd. 
Ereignisse vom 24. August zeigten erneut die Fragilität der christlichen Allianz und die Bedeutung der Etikette. An diesem Tag kehrte Admiral Rospigliosi mit einigen venezianischen Galeeren und dem maltesischen Verband von einer Exkursion zur Einholung von Trinkwasser zum Hauptankerplatz bei Koite zurück; die wartenden venezianischen Galeeren und Galeassen salutierten Rospigliosis capitana, aber nicht dem maltesische Flaggschiff mit den üblichen Kanonenschüssen. Daraufhin berief Generalkapitän Accarigi seinen Offiziersrat ein und informierte anschließend Rospigliosi, dass der maltesische Verband aufgrund dieser Brüskierung nach Malta zurückkehren werde. ${ }^{83}$ Rospigliosi konnte die Malteser nicht zum Bleiben zwingen, und für eine Anfrage zur Klärung der Affäre an die Römische Kurie blieb keine Zeit. ${ }^{84}$ Am 28. August gab Generalkapitän Accarigi tatsächlich den Befehl zur Abreise, und der maltesische Verband begab sich nach Messina, wo die Schiffe überwintern und überholt werden sollten.

Padre Ottomano blieb indes am Ort des Geschehens und verbrachte den Großteil seiner Zeit wechselweise auf Rospigliosis capitana und der Galeere San Pietro von Komtur Buontempi. Zur Erleichterung der Venezianer trafen Nachrichten vom baldigen Aufbruch eines spanischen Verbandes in Richtung des griechischen Archipels ein. Am 19. September trafen die Spanier vor Korfu ein, wo bereits einige venezianische Transportschiffe und leichte Kriegsschiffe warteten. Heraufziehende Stürme hielten die Schiffe dort jedoch fest, sodass nach einigen Tagen entschieden wurde, eine Weiterreise nach Kreta aufzugeben und die maritime Saison zu beenden. Der spanische Verband kehrte wieder nach Neapel zurück. Von diesen Stürmen wurde auch die nach wie vor an Kretas Südküste ankernde osmanische Transportflotte empfindlich dezimiert.

Unterdessen hatte sich Admiral Rospigliosi von Kreta nach Rom begeben, um seinem Onkel auf dem Heiligen Stuhl den Verlauf der Kampagne und das bisher enttäuschende Resultat der Präsenz des Padre Ottomano vor Kreta zu schildern.

Entgegen den Erwartungen war die Kampagne des Jahres 1668 trotz allem noch nicht beendet. Anfang November erreichte ein von François d'Aubusson, Duc de la Feuillade, geführter Verband von 800 Söldnern und etwa 400 freiwilligen Abenteurern, darunter viele französische Aristokraten, Kreta. ${ }^{85}$ De la Feuil-

83 Zur Problematik der Etikette and des Status vgl. auch de Daru, Geschichte der Republik Venedig (wie Anm. 12), S. 329-333.

84 Bigge, Der Kampf um Candia (wie Anm. 12), S. 150.

85 Zur Zusammensetzung des von de la Feuillade nach Kreta geführten Korps vgl. Anonym, Journal véritable de tout ce qui s'est passé en Candie sous le Duc de la Feuillade, Paris 1670; Anonym, Journal du l'Expédition de M. de Feuillade pour le secours de Candie, Lyon 1669; vgl. auch ausführlich Dominique Bouhours, Histoire de P. D'Aubusson-La Feuillade, Grand-Maître de Rhodes, Paris 1806, S. 510-525, und die Zusammenfassungen in Bigge, Der Kampf um Candia (wie 
lades Verband war - aus bereits beschriebenen Gründen - von Marseille nach Kreta unter der Flagge des Malteserordens gesegelt. Wenige Tage später trafen weitere 1600 venezianische und 400 französische Söldner unter dem Kommando des Comte d'Harcourt sowie ein maltesisches Bataillon mit 400 Söldnern und 63 Ordensrittern auf Kreta ein. ${ }^{86}$

In den Augen des Duc de la Feuillade waren diese Truppen ausreichend, um einen Überraschungsangriff auf die osmanischen Linien durchzuführen. Diese Überlegungen gingen weniger auf nüchterne Einschätzungen der Stärke und Möglichkeiten der vorhandenen Kräfte zurück, sondern scheinen vielmehr von Motiven wie Ruhmsucht bei den neu angekommenen Offizieren und Adeligen vorangetrieben worden $\mathrm{zu}$ sein. Diese hatten kein Interesse, weitere fünf Monate in der kalten Saison in den Kasematten und Gräben der ausgehungerten Festungsstadt zu verbringen und auf die Eröffnung der neuen Kampagne im Frühjahr 1669 zu warten. Darüber hinaus ließ der finanzielle Aspekt de la Feuillade auf rasche Aktionen drängen, immerhin hatte der Herzog die Hälfte der Kosten für Zusammenstellung und Transport seines Verbandes selbst übernommen und stand in der Verpflichtung der Lohnzahlung an die von ihm angeworbenen Söldner.

Als de la Feuillade General Francesco Morosini einen baldigen Beginn der Attacke vorschlug, lehnte Letzterer dies mit dem Verweis auf die limitierten Kräfte der Christen und die taktische Kompetenz des osmanischen Gegners strikt ab. ${ }^{87}$ De la Feuillade beharrte indes auf sein Vorhaben und erklärte, diesen Angriff dann eigenmächtig durchführen zu wollen. Aufgrund des Freiwilligenstatus von de la Feuillades Truppen konnte Morosini dieses nicht verbieten, untersagte aber die Beteiligung venezianischer Verbände. De la Feuillade terminierte seinen Ausfall auf den 16. Dezember und ungeachtet der ablehnenden Haltung General Morosinis erklärten auch 48 Malteserritter und das maltesische Bataillon ihre Bereitschaft, an dem Unternehmen teilzunehmen. ${ }^{88}$ Als der Angriff in den frühen Morgenstunden des 16. Dezembers begann, trug der Überraschungseffekt tatsächlich dazu bei, dass auf einem größeren Frontabschnitt die erste Linie und vorgelagerten Gräben der Belagerer überrannt werden konnten. Nachdem sich die

Anm. 12), S. 150; de Daru, Geschichte der Republik Venedig (wie Anm. 12), S. 335-337; Eickhoff, Venedig, Wien und die Osmanen (wie Anm. 2), S. 245-247.

86 Vgl. Bigge, Der Kampf um Candia (wie Anm. 12), S. $158 \mathrm{f}$.

87 Ebd., S. $160 \mathrm{f}$.

88 Zum Angriff von de le Feuillade vgl. ausführlich Anonym, Journal véritable (wie Anm. 85), S. 80-84; Anonym, Journal du l'Expédition (wie Anm. 85), S. 30-37; de Daru, Geschichte der Republik Venedig (wie Anm. 12), S. 338-340; Eickhoff, Venedig, Wien und die Osmanen (wie Anm. 2), S. 298. 
Osmanen jedoch gesammelt hatten, führten heftiges Artilleriefeuer und gezielte Gegenangriffe zum raschen Ende des Ausfalls, der Verband de la Feuillades und der Malteser wurde unter hohen Verlusten - gemäß den zeitgenössischen Quellen die Hälfte der Angreifer - wieder in die Ausgangsstellungen zurückgeworfen. De la Feuillades Interesse an einer Fortführung des Kampfes war erloschen. Der Herzog verließ mit dem Rest seines Verbandes am 24. Januar den Schauplatz der Niederlage und reiste nach Frankreich zurück. ${ }^{89}$

\section{Eine neue Front auf dem Balkan und in Griechenland?}

$\mathrm{Zu}$ diesem Zeitpunkt hatte auch Padre Ottomano Kandia verlassen. Die Quellen verraten nichts Näheres über die Abfahrt, das Schiff und eventuellen Begleitschutz; dokumentiert ist jedoch, dass der Einsatzplan des berühmten Dominikaners geändert wurde. Da seine Anwesenheit auf Kreta keinen Effekt auf die Angreifer hatte bzw. den feindlichen Truppen nicht umfassend und erfolgreich »medial« vermittelt werden konnte, erinnerte man sich nun der Vorschläge der 1667 nach Paris entsandten armenischen Emissäre und der brieflich dargelegten Agenda des Generalvikars der Griechisch-Orthodoxen Kirche von Alexandria und des Wallachenprinzen Gregor Basil Rákóczi. Nun wurde auf die Provokation einer Rebellion auf dem griechischen Festland und an der südlichen Küste des Balkans gesetzt. Damit sollte eine neue, die Wucht der osmanischen Angriffe auf Kandia ablenkende Front eröffnet werden..$^{90}$

Im Gegensatz zur Situation auf Kreta hoffte man auf dem Balkan und auf dem griechischen Festland, eine persönliche Kontaktaufnahme des Padre mit führenden Repräsentanten der Bevölkerung erreichen zu können. Nach wie vor setzte man auf die physische Präsenz des angeblich erstgeborenen Sohns von Sultan Ibrahim, um einen Aufstand gegen seinen jüngeren Bruder Mehmet IV., der den Thron durch »Usurpation« erklommen habe, zu erreichen. Dies konnte natürlich nur unter massivem Begleitschutz und unter Ausnutzung eines Netzes von Informanten und teilweise geheimen Reisewegen geschehen. Zuerst bestand die Absicht, den Padre nach Kythira zu bringen. Dieses Vorhaben wurde auf Rat von General Morosini wieder aufgegeben; stattdessen erfolgte der Transfer des

89 Bigge, Der Kampf um Candia (wie Anm. 12), S. $160 f$.

90 Charles Terlinden, Le Pape Clément IX et la guerre de Candie (1667-1669) d'après les Archives Secrétes du Saint-Siège, Paris 1904, S. 133; Callus, Il P. Domenico Ottomano (wie Anm. 10), S. 99. 
Dominikaners - immer in Begleitung seines auch des Griechischen mächtigen Sekretärs Ignozzi di Terracusa - zur Insel Zakyntos (in den zeitgenössischen italienischen Dokumenten »Zante $«) .{ }^{91}$ Hier trafen die beiden Ende November 1668 ein und präsentierten ihre von Morosini ausgestellten Empfehlungsschreiben und Anweisungen an den dortigen venezianischen Gouverneur Valier. ${ }^{92}$

Mit dessen Hilfe und der von einheimischen Handelsherren wurden in den folgenden Wochen Kontakte $\mathrm{zu}$ verschiedenen griechischen, albanischen, mazedonischen und bulgarischen Repräsentanten sowie anderen auf dem griechischen Archipel und südlichen Balkan lebenden ethnischen Gruppierungen aufgenommen. Gemeinsam beriet man, wie und wann ein allgemeiner Aufstand gegen die osmanischen Besatzer organisiert werden könnte. Die meisten dieser Repräsentanten versprachen loszuschlagen, sobald sie direkte militärische Hilfe und Lieferungen moderner Waffen erhalten hätten.

Im Dezember 1668 und im Januar des Folgejahres schrieb Padre Ottoman zu diesem Zweck zahlreiche Briefe an den Senat Venedigs, die Römische Kurie, den Großmeister von Malta und andere europäische Fürsten, um weitere und bessere Schusswaffen sowie logistische Hilfe zu erhalten. Als bis März keine Antwortschreiben auf diese Bitten in Zakyntos eintrafen, bat der Padre den damals auf seinem Weg von Kandia nach Venedig Zwischenstation auf Zakyntos einlegenden venezianischen Militär und Diplomaten Marchese Annibale Porrone um Unterstützung. Annibale Porrone sollte in Venedig auf raschen Beistand für das oben geschilderte Vorhaben dringen. Der Dominikanerpater legte noch einmal dar, welche großen Vorteile nicht nur für Venedig, sondern für alle christlichen Mächte im östlichen und zentralen Mittelmeer eine zweite Front auf dem südlichen Balkan und in Griechenland mit sich bringen würde.

Als Porrone Anfang April in Venedig eintraf, trug er dieses Anliegen sowohl dem Senat als auch dem apostolischen Nuntius Lorenzo Trotti, Erzbischof von Karthago, vor. ${ }^{93}$ Es dauerte allerdings bis Anfang Mai 1669, bis das Thema im venezianischen Senat diskutierte wurde. Die Mehrheit des Senats zeigte sich

91 Zum Aufenthalt und zu den Aktivitäten des Padre Ottomano auf Zakyntos vgl. Eickhoff, Venedig, Wien und die Osmanen (wie Anm. 2), S. 249; Dal Pozzo, Historia della Sacra Religione (wie Anm. 7), vol. 2, S. 353. Das Schreiben von Francesco Morosini an den venezianischen Senat vom 20.11.1668 (Archivio di Stato, Venezia, Dispaccio del capitano generale de Mare, fz. 31, n. 107) wird hier zit. nach Missak Effendi, Le Père Ottoman (wie Anm. 54), S. 375-377; vgl. auch Callus, Il P. Domenico Ottomano (wie Anm. 10), S. 99-102.

92 Vgl. die teilweise falsche Zusammenfassung bei Dal Pozzo, Historia della Sacra Religione (wie Anm. 7), vol. 2, S. 352f.

93 Vgl. Lorenzo Trottis Bericht vom 6.4.1669, ASV, Nunziatura di Venezia, Monsignor Nunzio al Cardinale Segretario di Stato, n. 108, hier zit. nach Missak Effendi, Le Père Ottoman (wie Anm. 54), S. 375-377. 
skeptisch gegenüber dem Vorschlag aus Zakyntos, einen umfassenden und vor allem erfolgreichen Aufstand in oben geschilderter Region zu provozieren. Im Übrigen seien die venezianischen Ressourcen für die Finanzierung eines solchen Aufstandes nach über zwanzig Jahren Kampf um Kandia zu erschöpft, um hier aktiv und offensiv vorgehen zu können. Ferner wurde das Ergebnis eines tatsächlich erfolgreichen Aufstands gegen die osmanischen Machthaber kontrovers diskutiert. Würden die Aufständischen nach einem Sieg der venezianischen Sache loyal gegenüberstehen und bereits bestehende Handels- und Transitabkommen respektieren? Bestand hier nicht die Gefahr, eine unberechenbare, instabile, von lang anhaltenden Stammes- und Bürgerkriegen geprägte Region zu schaffen?

Weitere Aspekte dieser um den Padre Ottomano kreisenden Idee eines Volksaufstandes gegen den regierenden osmanischen Sultan warfen für Venedig unkalkulierbare Probleme und Gefahren auf. Würden nicht benachbarte christliche Mächte, etwa Österreich, ein eventuell entstehendes Machtvakuum auf dem Balkan und an der adriatischen Küste für eigene Expansionsinteressen nutzen? Noch weit mehr als an den Häfen und Festungen Kretas musste die Serenissima an einer steten Verfügbarkeit der lebenswichtigen adriatischen Handelslinien und Seehäfen interessiert sein.

Aufgrund dieser Überlegungen lehnte der Senat schließlich die von Padre Ottomano bzw. seinem Umfeld gemachten Vorschlägen ab. Dies bedeutete, dass weiter Hoffnung auf die militärische Lösung des Problems auf Kreta durch fremde Entsatztruppen bestand. Vor allem seitens Frankreichs und Spaniens hoffte man für die Kampagne des Jahres 1669 auf eine Erweiterung der militärischen und logistischen Hilfe. ${ }^{94}$ Dabei ist spätestens $\mathrm{zu}$ diesem Zeitpunkt eine deutliche Diskrepanz zwischen der Lageeinschätzung des Senats bzw. der Regierungskreise der Serenissima einerseits und den vor Ort kämpfenden Offizieren und Verantwortlichen andererseits $\mathrm{zu}$ beobachten. Die umfassenden strategischen und globalen Konzepte der im fernen Venedig agierenden Regierungsvertreter ließen sich kaum mehr mit der von den drängenden Notwendigkeiten des Überlebenskampfes geprägten Wahrnehmung der Verteidiger Kandias vereinbaren. Letztere hätten mit Sicherheit die Eröffnung einer zweiten Front auf dem griechischen Festland als höchst willkommene Entlastung begrüßt. Leider geben die Archivalien keinen direkten Einblick in das persönliche Umfeld, in dem sich der Padre Ottomano damals auf Zakyntos bewegte und das ihn als Protagonisten der oben

94 Vgl. das Schreiben Lorenzo Trottis an Kardinal Rospigliosi vom 4.5.1669, ASV, Nunziatura di Venezia, Monsignor Nunzio al Cardinale Segretario di Stato, n. 108, hier zit. nach Missak Effendi, Le Père Ottoman (wie Anm. 54), S. 375f. 
skizzierten Vision, eine Rebellion anzustoßen, instrumentalisierte. Höchstwahrscheinlich wurde ihm die Agenda von Offizieren wie Vincenzo Rospigliosi vorgeschrieben.

Die Verfechter dieses Lösungsansatzes für einen Entsatz von Kandia gaben indes auch nach dem ablehnenden Bescheid aus Venedig nicht auf. Sie beauftragten nun den Malteserritter, Komtur von Mailand und Balí von San Giovanni a Mare von Neapel, Carlo della Lengueglia ${ }^{95}$ - ebenfalls ein alter Bekannter des Padre Ottomano -, den Plan eines Aufstandes gegen Mehmet IV. weiterzuverfolgen. In seiner Funktion als Offizier in dem auf Kreta kämpfenden maltesischen Bataillon und Komtur von Mailand begab sich Lengueglia Anfang Mai auf eine Italienreise, in deren Verlauf auch Venedig und Rom Station sein sollten. Die ihm vor seiner Abreise überantworteten Papiere und Dokumente enthielten schonungslose und nüchterne Ausführungen über die tatsächliche, äußerst bedrohliche Lage der Verteidiger Kandias.

Um detaillierte Instruktionen und weitere Hintergrundinformationen über die Situation auf dem Balkan zu erhalten, besuchte Lengueglia Padre Ottomano auf Zakyntos, von wo er Anfang Mai 1669 wieder Richtung Venedig aufbrach. Ende Mai hatte der Malteserritter in Venedig Gelegenheit, den Mitgliedern des venezianischen Senats die kritische Lage der Verteidiger von Kandia darzulegen. Gemäß dem von ihm gezeichneten Szenario stand ohne die Realisierung einer zweiten Front auf dem Balkan eine Übergabe Kandias zeitnah bevor. Gleichzeitig berichtete er von den Aktivitäten des Padre Ottomano auf Zakyntos. Aufgrund des Zeitdrucks würde nun nicht mehr nur finanzielle und waffentechnische Unterstützung der potenziellen Aufständischen genügen, auch christliche Truppen müssten zur Beförderung und Kontrolle dieses Aufstands umgehend an südadriatische Häfen verlegt werden.

In einem von Lengueglia mit sich geführten, aber in den von dem Autor des vorliegenden Beitrags gesichteten Archivalien nicht auffindbaren Memoire des Padre Ottomano war von 4000 benötigten Soldaten die Rede. Im gleichen Schriftstück wird berichtet, dass die Vertreter der Regionen und ethnischen Gruppen auf dem südlichen Balkan und auf dem griechischen Festland auf die Auszahlung hoher Geldsummen beharrten, bevor sie ihre Anhänger zu einer Rebellion gegen die Osmanen führen würden. ${ }^{96}$

$95 \mathrm{Zu}$ Carlo della Lengueglias Karriere im Malteserorden vgl. Dal Pozzo, Historia della Sacra Religione (wie Anm. 7), vol. 2, S. 481.

96 Auf die Existenz dieses Schriftstücks wird verwiesen im Brief von Lorenzo Trotti an Kardinal Giacomo Rospigliosi vom 18.5.1669, ASV, Nunziatura di Venezia, Monsignor Nunzio al Cardinale Segretario di Stato, n. 108, hier zit. nach Missak Effendi, Le Père Ottoman (wie Anm. 54), S. 376. Lorenzo Trotti skizziert eine kurze Zusammenfassung des Memoire. 
Lengueglias eindringliche Schilderungen verfehlten nicht ihre Wirkung. Anfang Juni 1669 wurden die Vorschläge aus dem Umkreis des Padre Ottomano erneut im Senat diskutiert. Das Resultat blieb jedoch negativ. Neben den oben genannten Gründen wurde nun auch auf die Unwägbarkeiten der Zahlung eines „Vorschusses« an die potenziellen Rebellenführer verwiesen. Die eingesetzten finanziellen Mittel böten keine Garantie für einen Erfolg des Unternehmens. ${ }^{97}$

Im Gegensatz zu den Verantwortlichen der Serenissima war der apostolische Nuntius in Venedig, Lorenzo Trotti, von der Stichhaltigkeit und Durchführbarkeit der von Lengueglia übermittelten Vorschläge überzeugt. In seiner Korrespondenz mit der Römischen Kurie folgte er rückhaltlos den Argumenten des berühmten Dominikanerpaters und schätzte eine finanzielle und militärische Intervention an der Küste des südlichen Balkans als »weder sehr gefährlich noch schwierig « ein. ${ }^{98}$ Währenddessen war Carlo della Lengueglia von Venedig weiter nach Rom gereist, wo er während einer Audienz bei Papst Klemens IX. Briefe des Padre Ottomano übergab. Doch auch in Rom sollten sich die Hoffnungen auf konkrete Hilfe für das Vorhaben des Padre und seiner Unterstützer nicht erfüllen. Dies mag angesichts des vorherigen energischen Eintretens des Papstes für die Venezianer und Verteidiger Kandias auf den ersten Blick überraschen.

Die Korrespondenz zwischen Kardinal Giacomo Rospigliosi und dem Nuntius in Paris, Niccolò Pietro Bargellini, vom Juli 1669 gibt einige Hinweise auf die tieferen Gründe der Römischen Kurie, eine direkte militärische Intervention oder einen Aufstand auf dem Balkan und in Griechenland nicht offen zu unterstützen. Die Briefe Rospigliosis und Bargellinis an Klemens IX. verweisen deutlich auf die Beunruhigung der französischen Regierung über die angeblichen »Verhandlungen zwischen dem Padre Ottomano und dem Großwesir« und die damals auf Zakyntos vorbereitete Entthronung Sultan Mehmets IV. ${ }^{99}$ Offensichtlich wurden die Rolle des Dominikanerpaters und seine Aktivitäten im fernen Zakyntos unterstützt durch christliche Propaganda und eine unklare Nachrichtenlage - in Paris massiv überschätzt.

Die französische Regierung sah eine Destabilisierung der politischen Verhältnisse auf dem Balkan und Griechenland äußerst skeptisch. Besonders wurde eine Intervention bzw. ein Ausnutzen dieser Instabilität durch die damals über

97 Vgl. Bigge, Der Kampf um Candia (wie Anm. 12), S. 152.

98 Vgl. das Schreiben von Lorenzo Trotti an Kardinal Rospigliosi vom 15.6.1669, ASV, Nunziatura di Venezia, Monsignor Nunzio al Cardinale Segretario di Stato, vol. 196, ff. 8v, hier zit. nach Missak Effendi, Le Père Ottoman (wie Anm. 54), S. 376f.

99 Vgl. die Schreiben vom apostolischen Nuntius in Paris, Bargellini, an Kardinal Rospigliosi vom 5. und 19.7.1669, ASV, Nunziatura di Francia, vol. 137, f. 692, ff. 692-695 und ff. 705-707, hier zit. nach Missak Effendi, Le Père Ottoman (wie Anm. 54), S. 376-378. 
Spanien und Österreich regierenden Habsburger gefürchtet. Gegenüber Nuntius Bargellini äußerte Ludwig XIV. seinen Unmut über die Nachrichten aus dem östlichen Mittelmeer. An einem diplomatischen Konflikt mit Frankreich konnte die Römische Kurie kein Interesse haben. ${ }^{100}$ Ende Juli und erneut am 13. August beeilte sich die Kurie, die französische Regierung $\mathrm{zu}$ informieren, dass es bisher weder $\mathrm{zu}$ »negotii gravi« zwischen Padre Ottomano und Großwesir Ahmet Köprülü gekommen sei, noch kommen werde. Es wurde ferner darauf verwiesen, dass die Aktivitäten des Dominikaners auf Zakyntos und dem griechischen Festland nicht auf Anordnung des Papstes erfolgen. ${ }^{101}$

Diese Hintergründe der päpstlichen Absage an die Hilfsgesuche des Padre Ottomano bzw. Carlo de Lengueglias konnten naturgemäß nicht offen formuliert werden. Dem Malteserritter wurde daher im August 1669 mitgeteilt, die Pläne des Padre würden einer soliden und realistischen Basis entbehren und hätten keine Chance auf Verwirklichung.

Am Ende waren es also die ökonomischen und strategischen Interessen Frankreichs und auch Venedigs selbst, die den Projekten des Padre Ottomano und seines Umfelds ein Ende setzten. Dabei steht dahin, ob diese erfolgreich verlaufen wären oder nicht. Die Vorstellung, dass der Dominikanerpater nominell über ein sich gegen den Sultan erhebendes Griechenland herrsche - wie es für den begrenzten Rahmen seines Einsatzes zum Entsatz Kandias gewünscht bzw. toleriert wurde -, war für Frankreich und auch andere Anrainerstaaten keine Option zum bisherigen Modus Vivendi.

Dieser Mangel einer unitas christiana wurde von zeitgenössischen katholischen Biografen des Padre Ottomano wie Ottaviano Bulgarini, Jacques de Jant oder Felice Tempia, die jenen als einen an den materiellen Interessen der Europäer gescheiterten christlichen Helden präsentieren, wortreich beklagt. Ihr Protagonist erscheint als ein Charakter, »che appare vittima destinato di uno scontro storico più forte di lui, e che cerca invano di accordare le armi cristiane nelle sforzo commune contro il paese da cui era uscito fanciullo «. ${ }^{102}$

100 Robert Darricau, Une heure mémorable dans les rapports entre la France et le Saint-Siège: le pontificat de Clément IX. In: Bulletino di Storia Pistoiese, 71 (1969), S. 73-98; Georg Lutz, Zur Papstfinanz von Klemens IX. In: Römische Quartalsschrift, 74 (1979), S. 32-90.

101 Vgl. die Schreiben von Kardinal Rospigliosi an Pietro Bargellini vom 30.7. und 13.8.1669, ASV, Nunziatura di Francia, vol. 137, ff. 154-157 und 160f., abgedruckt in Terlinden, Le Pape Clément IX (wie Anm. 90), S. 185-187; vgl. auch Missak Effendi, Le Père Ottoman (wie Anm. 54), S. 376; Callus, Il P. Domenico Ottomano (wie Anm. 10), S. 110.

$102 »[$ Padre Ottomano] erscheint als Opfer von historischen Auseinandersetzungen, die sein Schicksal bestimmten; eine Person, die vergeblich versucht, die christlichen Waffen gegen das Land zu vereinen, welches er als Kleinkind verlassen hatte.« Franco Lanza, La Narrativa del 


\section{Moderne Literaturhistoriker haben dementsprechend analysiert: \\ »Il padre Ottomano raccoglie l'idealità cavalleresca in quanto missione sacrificale: è dunque un eroe passive, incurante del ricatto politico a cui lo espongono i potenti, preoccupato del bene altrui assai più che della propria gloria. $«^{103}$}

Es muss daher auch für die englische und niederländische Seite eine Erleichterung gewesen sein zu erfahren, dass die Mission des Padre Ottomano 1668 und 1669 kaum einen direkten Effekt auf die Situation im östlichen Mittelmeer, deren Stabilität und Status Quo erkennen ließ. Als im Sommer 1669 von christlicher Seite keinerlei positive Signale auf aktive, materielle Unterstützung des Dominikanerpaters zu erkennen waren, verschwand dessen schillernde Figur auch aus dem öffentlichen Diskurs des theatrum europaeum. ${ }^{104}$

Ende September verließ er Zakyntos in Richtung Venedig. Dort traf er am 10. Oktober ein und nahm im Haus seines Vertrauten Carlo de Lengueglia Quartier. ${ }^{105}$ Obwohl es seinem Biografen Bulgarini zufolge bald zu einem Treffen mit dem venezianischen Senat kam, wo der Dominikaner Gelegenheit hatte, über die Ereignisse in Kandia und Zakyntos ausführlich $\mathrm{zu}$ referieren, konnten bisher keine entsprechenden, dieses belegende Archivalien gefunden werden. Von nun an nahm der Dominikaner erneut seinen zurückgezogenen Lebensstil an.

Sich mit den letzten Lebensjahren des Padre Ottomano zu beschäftigen, ist für das Thema des vorliegenden Beitrags nicht von Belang. Es soll lediglich angemerkt werden, dass der Dominikaner nach fünf Jahren Aufenthalt in Rom wieder nach Malta übersiedelte, wo er als Generalvikar der Dominikaner für die Insel am 26. Oktober 1676 im Alter von 34 Jahren im Konvent von Porto Salvo an der Pest starb. ${ }^{106}$

Cavalieri Gerosolimitani. In: La più stupenda e gloriosa macchina. I romanzo italiano del secolo XVII. Ed. di Mario Santoro, Neapel 1981, S. 95.

103 »Hinsichtlich einer von Selbstopfer geprägten Mission vereinigt der Padre Ottomano die Idealvorstellung des Rittertums; er ist insofern ein passiver Held, sorg- und wehrlos gegenüber politischen Erpressungen der Mächtigen, mehr mit Interessen anderer als mit dem eigenen Ruhm beschäftigt.«Ebd.

104 Vgl. die im Alten Reich sehr populäre Anthologie mit dem programmatischen Titel von Erasmus Francisci, Theatrum Europaeum, Nürnberg 1690.

105 Vgl. den Brief des apostolischen Nuntius in Venedig, Lorenzo Trotti, an Kardinal Rospigliosi vom 12.10.1669, ASV, Nunziatura di Venezia, vol. 108; hier zit. nach Callus, Il P. Domenico Ottomano (wie Anm. 10), S. 119.

106 Liber Mortuorum S. Mariae Portus Salutis, 1650-1694, f. 161, hier zit. nach Callus, Il P. Domenico Ottomano (wie Anm. 10), S. 129. 


\section{Resümee}

Der Krieg um Kreta war zum Zeitpunkt des Todes des Padre Ottomano seit Langem beendet. Denn er endete bereits - nach dem Ausbleiben schlagkräftiger Entsatztruppen $^{107}$ - am 6. September 1669 mit der venezianischen Kapitulation. ${ }^{108}$ Wenig später wurde ein osmanisch-venezianisches Friedensabkommen geschlossen, das von Francesco Morosini anscheinend ohne Autorisierung durch den Senat unterzeichnet wurde; ein pragmatisches Vorgehen, das weiteren Verlust an Truppen und Material verhinderte. ${ }^{109}$ Das später vom Dogen ratifizierte Abkommen erlaubte ferner, im Besitz der Festungen bzw. Häfen von Gramvousa, Spinalonga und Souda zu bleiben. Damit war der Schutz für den fortgesetzten Handel Venedigs mit der Levante gewährleistet. Darüber hinaus wurde die Lagunenrepublik für den Verlust von Kreta mit Stützpunkten an der dalmatinischen Küste entschädigt.

Die Rolle des Padre Ottomano in den letzten Kampagnen des Krieges um Kandia steht daher im Spannungsfeld eines von der Gegenreformation, militanter Missionsarbeit und anti-osmanischer Propaganda geprägten katholischen Geistes sowie eines politischen und vor allem ökonomischen Pragmatismus. Sein Agieren auf Zakyntos und vor allem sein letztliches Scheitern, eine zweite Front auf dem südlichen Balkan und griechischen Festland aufzubauen, lässt sich nur im Kontext des seit Langem manifesten französisch-habsburgischen Antagonismus verstehen. Für Frankreich - und auch für England und die Niederlande - war eine Destabilisierung dieser Region und eine Veränderung des Status Quo nicht wünschenswert. Die Installation eines unter der geistigen Führung des Papstes stehenden angeblichen älteren Bruders des regierenden Sultans Mehmet IV. als Kronprätendent und nominellen Herrscher über ein christliches Reich im östlichen Mittelmeer lag nicht in ihrem Interesse. Die Antwort auf die Frage, ob dies mit massiver okzidentaler Hilfe möglich gewesen wäre, muss Spekulation bleiben. Fakt ist, dass bereits der strategische Einsatz des Padre Ottomano zum Entsatz von Kandia ein eindeutiger Fehlschlag war.

107 Zur Kampagne von 1669 vgl. Registro di lettere del Cav. F. Francesco Rospigliosi, ASV, miscell., arm. XVI, vol. 138; Richard Reaux de la Richardière, Le Voyage de Candie fuit par l'Armée de France en l'Année 1669, Paris 1681.

108 Zur Kampagne von 1669 und der Kapitulation vgl. Greene, A Shared World (wie Anm. 2), S. 56; Anderson, Naval Wars in the Levante (wie Anm. 3), S. 181-185.

109 Zur letzten Phase des Krieges vgl. Eickhoff, Venedig, Wien und die Osmanen (wie Anm. 2), S. 259-266; Bigge, Der Kampf um Candia (wie Anm. 12), S. 187-192. 